Volume 12 Number 3, July-September 2018: pp. 243 - 283. Copyright (c) 2018 FIAT JUSTISIA. Faculty of Law, Lampung University, Bandarlampung, Lampung, Indonesia. ISSN: 1978-5186 | e-ISSN: 2477-6238.

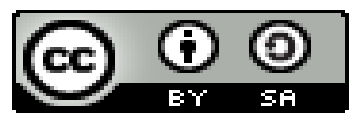

\title{
La responsabilité pénale des mineurs en droit international et en droit congolais : Entre un pragmatisme justifié et un dogmatisme affirmé
}

\author{
Bienvenu Wane Bameme \\ Université de Kinshasa/RDC, Democratic Republic of the Congo \\ wanebameme1@gmail.com \\ Ghislain-David Kasongo Lukoji \\ Université de Kinshasa/RDC, Democratic Republic of the Congo \\ ghislainlukoji@gmail.com
}

\section{Résumé}

En droit international, comme en droit interne congolais, le traitement des personnes âgées de moins de dix-huit ans en matière pénale, interpelle et inquiète. L'interpellation se rapporte particulièrement à la situation de l'enfant accusé d'avoir accompli un acte infractionnel; et l'inquiétude est suscitée notamment par rapport au traitement de l'enfant prétendant avoir subi un préjudice à la suite de l'infraction. L'étude a d'abord utilisé la méthode exégétique, en recherchant le vrai sens à donner aux dispositions normatives en rapport avec la question sous examen. Elle a recouru ensuite à la méthode comparative, en rapprochant en effet de la réglementation internationale, celle congolaise. S'il est vrai que d'une part, la réflexion a souligné la justification pragmatique du droit international de la responsabilité pénale des mineurs, à travers différents instruments internationaux, régulièrement conclus et justement appliqués; il n'est pas moins vrai qu'elle a noté d'autre part, l'infirmation purement dogmatique de la responsabilité pénale des mineurs de dix-huit ans en droit de la République démocratique du Congo. Il est à retenir qu'en réalité, le clivage mineurmajeur, cesse d'être celui de responsabilité pénale totale et irresponsabilité 
pénale absolue ou de capacité-incapacité, pour ainsi laisser place à celui de capacité spéciale-capacité générale.

How to cite: Bienvenu Wane Bameme and Ghislain-David Kasongo Lukoji, "La responsabilité pénale des mineurs en droit international et en droit congolais : Entre un pragmatisme justifié et un dogmatisme affirmé", Fiat Justisia, 12 (3), (2018).'

DOI: https://doi.org/10.25041/fiatjustisia.v12no3.1373

Mots-clés : Droit Penal International, Droit Congolasi, Responsabilite Penal, et Droit International

\section{A. Introduction}

En date du 31 décembre 2015, la loi n ${ }^{\circ} 15 / 022$ modifiant et complétant le décret du 30 janvier 1940 portant Code pénal a été promulguée. L'une des dispositions de ce texte insère, dans ledit code, 1 'article 20 ter fixant la majorité pénale à dix-huit ans. D'après une certaine lecture, le législateur congolais consacrerait, à travers cette disposition, une irresponsabilité pénale des mineurs pour les crimes internationaux, à la suite, à la fois des règles internationales en la matière et du principe d'irresponsabilité pénale affirmé depuis bien longtemps en droit interne congolais. A cet effet, l'intitulé de la présente réflexion paraitrait pour le moins incongru. Le lecteur pourrait donc s'interroger pour savoir s'il ne serait pas absurde de prétendre à une responsabilité pénale en droit international des individus, dès lors que l'existence même de celle de droit interne prête à débat ?

Mais, à ce dernier, nous nous employons à épingler les insinuations essentielles caractérisant mais surtout clarifiant la matière, sous une autre lecture.

D'entrée de jeu, il importe de rappeler ce qu'il faut entendre par “ responsabilité". En effet, ce mot se rapporte à l'adjectif "responsable"et peut avoir une double signification. La première se rapporte à l'aptitude d'agir et désigne celui qui est capable de prendre une décision réfléchie en pesant les conséquences de ses actes; la seconde se réfère aux conséquences et désigne celui qui doit répondre de ses actes ou réparer une faute ${ }^{1}$. Les deux facettes sont souvent liées ; mais c'est la seconde qui induit traditionnellement l'acception juridique. Ainsi, la responsabilité juridique peut être définie comme "la qualité de ceux qui doivent [...], en vertu d'une règle, être choisis

\footnotetext{
${ }^{1}$ R. Nerac-Croisier, “Irresponsabilité ou responsabilité ?", in R. NERAC-CROISIER (dir), Le Mineur et le droit pénal, Paris: L’Harmattan, (1997), pp.133-148.
} 
comme sujets passifs d'une sanction"2. Elle est dite " civile » lorsque l'individu est appelé à réparer le préjudice causé à autrui par ses actes; ; et "pénale", lorsqu'il est obligé de subir l'une ou des sanctions pénales du fait de ses actes infractionnels.

La question qu'il sied, alors, de se poser est celle de savoir s'il existe des règles de droit qui appellent les mineurs à répondre des faits infractionnels dont ils viendraient à être auteurs. Au cas contraire, quels seraient les mécanismes prévus à leur égard, quelles en serait la nature ?

Pour y répondre, nous analyserons d'abord les règles de la responsabilité pénale de cette catégorie de personnes en droit international (I), lequel est souvent appelé en garantie par la doctrine dominante, pour justifier la thèse de l'irresponsabilité des mineurs, avant de traiter des règles du droit interne congolais (II) en la matière.

\section{B. Discussion}

\section{Le droit international de la responsabilite penale des mineurs : Un pragmatisme justifié}

Notons d'emblée que la quasi-totalité des règles internationales relatives aux mineurs n'abordent que très rarement les aspects pénaux. Et même, celles qui saisissent la question, se rapportent dans la majeure partie aux mineurs victimes d'infractions (ou d'autres abus), et subsidiairement, aux garanties procédurales dont doivent bénéficier les mineurs mis en cause. Ce mutisme n'est pas seulement dû au défi cornélien ${ }^{4}$ (protection versus répression) qui caractérise la justice des mineurs, mais aussi et surtout à la politique dite criminelle, ou plutôt anticriminelle prônée par les instances internationales.

En effet, le mineur a émergé sur la scène internationale, avant tout, comme un sujet vulnérable. Il est donc apparu premièrement comme une victime principale de l'atrocité humaine et des calamités naturelles. De sorte qu'il s'est avéré inopportun et paradoxal de penser, en même temps, à des règles juridiques instituant sa responsabilité pénale.

Mais, cette noble posture, ayant laissé libre action aux droits internes, a été depuis peu revue. L'on a commencé à assister, crescendo, à l'encadrement par

\footnotetext{
${ }^{2}$ P. Fauconnet cité par J. Pradel, Droit pénal général, Cujas, 19 ème éd., Paris, (2012), p.379.

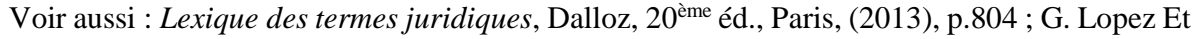
S. Tzitzis (dir.), Dictionnaire des sciences criminelles, Paris : Dalloz, (2004), pp. 832-836. ${ }^{3}$ Définition générique qui écarte volontiers le cas de la responsabilité pour fait d'autrui.

${ }^{4} \mathrm{~J}$. ZermatteN, "La loi fédérale régissant la condition pénale des mineurs", in Chronique de l'AIMJF, 1 (13), (2004), pp. 11-16, spéc.2-3.
} 
le droit international des droits de l'homme, des législations internes de plus en plus diversifiées (A), et à la régulation de certaines situations spécifiques de la vie internationale du mineur concernant particulièrement sa participation aux hostilités (B).

\section{La responsabilité pénale des mineurs en droit international des droits de l'Homme (DIDH) : des règles de Beijing à la Convention Internationale des Droits de l'Enfant (CIDE)}

L'encadrement des législations nationales relatives à la délinquance des mineurs s'est opéré par le truchement du Droit international des Droits de l'Homme, DIDH en sigle. Après les jalons jetés par les déclarations de 1924 et $1959^{5}$, l'enfant ${ }^{6}$, entendu ici comme mineur, et donc pris dans le sens d'une personne de bas âge (selon les Etats), ne constituera plus un objet particulier des Conventions internationales (sauf en matière d'adoption) jusqu'au milieu des années $80^{7}$. Malgré ce regain d'intérêt pour l'enfance et la place de choix accordée aux mineurs contrevenants ${ }^{8}$ dans ces Conventions, la question de leur responsabilité pénale ne sera toujours pas sujette à des règles claires et explicites.

Son appréhension appelle constamment une analyse transversale de plusieurs textes dont deux particulièrement : les règles de Beijing et la Convention internationale des droits de l'enfant (CIDE).

\footnotetext{
${ }^{5}$ La Déclaration sur les droits de l'enfant (SDN, 26/09/1924) et la Déclaration des droits de l'enfant (Résolution 1386, XIV, ONU/A.G, 20/11/1959).

${ }^{6}$ La Convention Internationale des Droits de l'Enfant, CIDE en sigle. Convention des NationsUnies du 20 novembre 1989. Article premier : “Au sens de la présente Convention, un enfant s'entend de tout être humain âgé de moins de dix-huit ans, sauf si la majorité est atteinte plus tôt en vertu de la législation qui lui est applicable".

${ }^{7}$ Laquelle époque coïncide avec la remise en cause généralisée du modèle protectionnel (concept développé supra).

${ }^{8}$ Ensemble de règles minima des N.U concernant l'administration de la justice pour mineurs (“Règles de Beijing”, Rés. 40/33, NU/AG, 29/11/1985) ; Règles minima des N.U pour l'élaboration de mesures non privatives de liberté ("Règles de Tokyo", Rés. 45/110, NU/AG, 14/12/1990) ; Principes directeurs des N.U pour la prévention de la délinquance juvénile (“Principes de Riyad », Rés. 45/112, NU/AG, 14/12/1990) ; Règles des N.U pour la protection des mineurs privés de liberté (" Règles de La Havane », Rés. 45/113, NU/AG, 14/12/1990) ; Résolutions 1996/13 du 23/07/1996 et 1997/30 du 21/07/1997 du CES relative à l'Administration de la justice pour mineurs ; Directives relatives aux enfants dans le système de la justice pénale ("Directives de Vienne", Rés. 1997/30, NU/CES, 25/02/1997) ; Observation $n^{\circ} 10 / 2007$ sur les droits des enfants dans la justice pour mineurs (CRC/C/GC/10, NU/CDE, 25/04/2007).
} 
Précurseur, le premier texte porte l'essentiel du régime juridique international applicable aux mineurs. Il est basé sur des standards minima de protection spécifique essentiellement judiciaire, élevés au rang des droits fondamentaux.

Successeur, le second quant à lui, sans apporter des innovations majeures de fond, a renforcé la juridicité de ce régime, notamment par le biais du principe de l'intérêt supérieur de l'enfant (ISE).

De ces instruments juridiques internationaux, il se dégage la non prohibition et donc la consécration de la responsabilité pénale du mineur triplement caractérisée d'abord dans une affirmation, ensuite dans une spécification, et enfin dans une relativisation.

\section{a. Une responsabilité pénale affirmée}

De la lecture des deux textes internationaux ci-dessus, il ressort la consécration d'une responsabilité pénale incontestée du mineur. En effet, ces instruments juridiques internationaux reconnaissent que l'enfant peut " être suspecté, accusé ou convaincu d'infraction à la loi pénale" et, de ce fait, “ répondre, au regard du système juridique considéré, de son délit" ${ }^{\prime 10}$ en subissant notamment des mesures privatives de liberté ${ }^{11}$.

Il n'y a aucun détour dans cette consécration de la responsabilité pénale du mineur au niveau international. Puisqu'on considère que la responsabilité pénale suppose que l'agent qui aura réalisé un comportement infractionnel, subisse à cet effet la sanction pénale y rattachée. Dès lors, ces instruments admettent à ce niveau international, qu'un mineur peut non seulement être suspecté, mais aussi accusé d'avoir commis une infraction prévue par un texte pénal. Il peut également être convaincu de cette infraction, c'est-à-dire que les enquêtes conduites et l'instruction menée dans le cadre d'une infraction donnée peuvent aboutir à ce que cela soit mis à sa charge. De ce qui précède, ce mineur fautif peut enfin répondre, c'est-à-dire supporter les conséquences de son comportement, en subissant entre autres des mesures de privation de liberté. Ces deux textes n'excluent donc pas la possibilité pour un mineur d'être tenu de subir par exemple la sanction pénale consistant en un enfermement.

\footnotetext{
${ }^{9}$ Article 40 de la Convention Internationale des Droits de l'Enfant, CIDE en sigle.

${ }^{10}$ Article 2.2 des Règles de Beijing.

${ }^{11}$ La Résolution 45/113 adopte une définition large et pragmatique des mesures privatives de liberté (11.b) englobant la phase pré-juridictionnelle (détention préventive) et juridictionnelle (jugement définitif de condamnation).
} 
Mais, il faut reconnaître que selon les textes, différents autres termes peuvent être usités en lieu et place de celles utilisés à l'égard des majeurs en visant un objectif particulier. C'est notamment ce qui fait la spécificité de cette responsabilité pénale.

\section{b. Une responsabilité pénale spécifique}

Une responsabilité visant le relèvement du mineur. Ces instruments juridiques internationaux évoqués rappellent la nécessité de distinguer les modalités d'élaboration et de mise en æuvre de cette responsabilité, dont l'objectif principal est le relèvement du mineur, et non la rétribution par sa punition. Ils se fondent sur le principe de l'intérêt supérieur ou du bien-être de l'enfant ${ }^{12}$ qui met à charge des Etats deux sortes d'obligations, à savoir : les obligations positives, consistant à réaliser pour l'enfant et à le protéger ; ainsi que les obligations négatives, exigeant le respect de l'enfant.

Appliqué à la responsabilité pénale, ce principe oblige d'une part, le législateur à prévoir un régime pénal spécifique qui respecte les droits, la dignité et les besoins des mineurs ${ }^{13}$. Il oblige d'autre part, le juge à prendre en compte non seulement la gravité du délit, mais aussi et surtout, les circonstances personnelles du mineur, auteur des faits, notamment sa position sociale, sa situation familiale, les dommages causés ou d'autres facteurs qui influent sur ces circonstances. Ces exigences se traduisent généralement aujourd'hui par une spécificité dans la norme pénale substantielle, caractérisée soit par l'atténuation des sanctions pénales, soit par l'autonomisation sanctionnelle purement et simplement. C'est que le texte pénal peut prévoir

\footnotetext{
${ }^{12}$ Le droit pénal met en ballotage plusieurs intérêts légalement protégés. Il s'efforce de réaliser un juste équilibre entre les droits des délinquants, les droits des victimes et les préoccupations sociétales relatives à la sécurité publique et à la prévention de l'infraction. En matière d'enfance délinquante, une préoccupation supplémentaire est prise en considération : le bien-être (ou l'intérêt supérieur) de l'enfant. Cette dernière, qui se veut comme la plus haute, doit être privilégiée au cas où elle entrerait frontalement en opposition avec d'autres intérêts légalement protégés. Lire avec intérêt T. HAMMABERG, “Le principe de l'intérêt supérieur de l'enfant : ce qu'il signifie et ce qu'il implique pour les adultes ", Allocution à la Conférence de Varsovie, 30/05/2008, Conseil de l'Europe, Commissariat aux droits de l'homme, Comm $\mathrm{DH} /$ Speech(2008)10 ; [en ligne] https://wcd.coe.int/ViewDoc. jsp?id=1313889\&Site ; G.-D. KASONGO LUKOJI, Existe-t-il un droit fondamental de l'enfant au respect de son intérêt supérieur? Etude comparée des droits anglais, belge et français, Mémoire de Master 2, DPA/Droits Fondamentaux, AMU, 2012, (Dir.) G. Scoffoni et P. Bonfils, pp. 11-14, 109 et ss. ; Voir l'article $14 \S 4$ du PIDC.

${ }^{13}$ V. Melnic, “La responsabilité pénale des mineurs dans le droit européen”, Révista, (2010), pp. 3-4,
} 
contre le mineur des sanctions pénales allégées par rapport à celles existantes ou des sanctions pénales nouvelles, et donc non prévues pour les majeurs.

Ces exigences se traduisent aussi par une spécificité dans la norme pénale formelle, renfermant pour cela une particularité liée à la procédure à côté d'une autonomisation institutionnelle. Il s'agit à ce niveau, d'un particularisme purement processuel, pouvant se manifester à travers diverses limitations, notamment, dans l'exigence du huis-clos et la restriction de la publicité...etc., ou plus directement par la création des juridictions spécialisées propres aux mineurs appliquant des règles de procédures adaptées aux mineurs.

Comme soutenu précédemment, le principal objectif visé est le relèvement du mineur. Mais, la réalisation d'un tel objectif a fait émerger d'autres catégories des mineurs dont la prise en compte s'est avérée, au demeurant, nécessaire au regard tant de l'interdépendance que de l'indivisibilité des droits de l'enfant (article 10 Rés. 1997/30) ainsi que des impératifs de prévention. Il existe un lien étroit entre tous les groupes des mineurs du fait que la quasi-totalité du contingent des mineurs-délinquants est fournie par les mineurs déviants et en danger. Ce faisant, les règles onusiennes ont vocation à s'appliquer à tous ces mineurs, y compris aux jeunes-adultes délinquants, et cela, selon la limite d'âge fixée dans chaque cas, à l'égard de qui sont prononcés des mesures de protection et d'aide sociale (article 3.1; 3.2-3.3 Rés. 40/33).

Ces règles tentent alors de concilier deux procédés pénaux apparemment antipodiques mais réellement complémentaires, à savoir : l'intervention antedelictum et la diversion (ou déjudiciarisation). Le premier est une action sur les facteurs criminogènes en vue de prévenir cette délinquance ${ }^{14}$; le second est un mécanisme de substitution au procédé judiciaire classique qui tend à extraire le plus possible le mineur du circuit pénal, quitte à le garder le plus longtemps possible sous l'empire socio-éducatif, et donc dans un cadre extrajuridictionnel.

14 Les interventions de la justice pour enfants s'orienteront non seulement vers les comportements qui violent le code pénal mais aussi certains actes estimés pré-délinquantiels (appelés aussi "actes déviants", “délits d'état"). Les Principes de Ryad en proposent une approche complète et positive impliquant tous les acteurs sociaux : la famille, l'école, la communauté, les médias, l'administration publique et l'Etat. La prévention y est présentée non pas comme une façon de s'attaquer à des situations négatives, mais comme un moyen de promouvoir l'intérêt général. 
Mais il n'exclue pas non plus une certaine possibilité de responsabilisation du mineur, surtout lorsqu'il inclut la médiation ${ }^{15}$. "Le recours aux services classiques de contrôle social s'érige en dernier ressort ${ }^{16}$. Sous cet angle, le Droit International des Droits de l'Homme, DIDH en sigle, se rapproche du modèle dit protectionniste.

\section{Une responsabilité pénale caractérisée par une terminologie adaptée au} mineur. On préfère à cet effet les expressions " enfants en conflits avec la loi » ou " enfants en contact avec la justice », en lieu et place de " délinquance juvénile », de " mineurs criminels », ou d" enfants délinquants », au motif pour certains que ces dernières expressions seraient stigmatisantes, et auraient des effets pernicieux pouvant mettre à mal la réinsertion du mineur, pourtant véritable auteur des faits infractionnels. Dans la même lignée, on propose la substitution terminologique, désignée par la doctrine de "déqualification pénale », des termes “infraction» et “peine» par “ manquement» et " mesure ".

Mais, il importe de souligner que rien ne peut justifier qu'une telle consécration d'une terminologie spécifique ou encore la restriction de la publicité de procédure, notamment en ce qui concerne le secret de l'identité du mineur et le huis-clos, parviennent à elles seules à suffire, pour prétendre à une irresponsabilité pénale des mineurs, en dehors d'une décision expresse du législateur concerné.

Il est donc plus qu'impérieux de limiter la portée de cette consécration terminologique, qui n'a jamais su s'imposer, à son champ originel qui est l'encadrement de la publicité de procédure dans un objectif purement réinsertionnel ${ }^{17}$.

A dire vrai, cette spécificité terminologique n'a pas abondamment attiré l'attention des instruments juridiques internationaux ${ }^{18}$. Il faut par contre

\footnotetext{
${ }^{15}$ Lire, M.J. Berbuz-Neneitez, "Sens et contre-sens du processus de médiation pénale dans la justice des mineurs. L'exemple espagnol à l'aune de la nouvelle loi organique", Journal du droit des jeunes, $8 / 2001, \mathrm{n}^{\circ} 208$, pp. 35-39

${ }^{16} \mathrm{M}$. Torelli, "Introduction à la protection internationale des droits de l'enfant”, CERDIRI, 2005, p.9.

${ }^{17}$ Article 1 Obs. $\mathrm{n}^{\circ}$ 10, 2007; articles 8 et 21 Résolution 40/33.

18 Cette terminologie n'a attiré à l'origine qu'une seule fois l'attention du législateur conventionnel (art. 1.3 et commentaire de l'art.3). Et, jusqu'à ce jour plusieurs textes internationaux, notamment l'article 40 de la CIDE, y compris le rapport du Comité des droits de l'enfant de 2007 qui reste le dernier état de lieu complet du droit onusien en cette matière,
} 
affirmer que jusqu'à ce jour, plusieurs textes internationaux, y compris le rapport du Comité des droits de l'enfant de 2007 qui reste le dernier état de lieu complet du droit onusien en cette matière, portent encore la terminologie classique, à savoir : “ infraction », " instruction », " poursuite », " coupable », “ âge de la responsabilité pénale », " incarcération » ...etc. ${ }^{19}$.

Mais, peu importe la terminologie consacrée, l'appellation retenue dans le texte ou la qualification réservée au processus de prise en charge des mineurs auteurs, coauteurs ou complices à l'acte infractionnel ainsi que ses modalités d'application ; le simple fait que la norme posée oblige les mineurs convaincus d'un comportement incriminé par la loi, à en assumer la charge en subissant la sanction pénale, c'est-à-dire la sanction qui afflige et s'exécute au nom de la société, rendant par conséquent ces mineurs, redevables vis-à-vis de la société, nous semble extrêmement pertinent, à défaut d'être particulièrement suffisant, pour admettre une responsabilité pénale des mineurs, bien qu'étant dans ce cas adaptée et donc spécifique à leur situation.

\section{c. Une responsabilité pénale relative}

Une relativité caractérisée par l'admission et la fixation du seuil d'âge de responsabilité pénale. Le point culminant d'un droit pénal des mineurs ${ }^{20}$ est l'admission et la fixation d'un (des) seuil(s) de responsabilité pénale. Si l'on se convient que la minorité pénale implique le droit à un traitement pénal différencié, c'est-à-dire ne pas être traité comme un adulte en matière pénale, et spécifique, ce qui voudrait renvoyer au fait d'être traité dans et pour son intérêt spécifique; elle constitue à cet effet, "non pas une cause de non imputabilité, mais une limite subjective et donc personnelle à l'application du code pénal et une ouverture à l'application du droit spécifique $»^{21}$. Dès lors, deux principaux seuils s'invitent au débat : d'une part, un seuil inférieur, ou la limite à partir duquel l'individu commence à bénéficier d'un tel régime

portent encore la terminologie classique (“Instruction”, "poursuite”, “infraction”, "âge de la responsabilité pénale", etc).

${ }^{19}$ Articles 37 et 40 de la Convention internationale des droits de l'enfant du 20 novembre 1989 ; 18 de la Charte africaine de la jeunesse du 2 juillet 2006 ; ...etc.

${ }^{20}$ Pour une certaine école, l'on ne peut pas parler d'un "droit pénal” à l'égard des mineurs étant donné que ces derniers seraient pénalement irresponsables. Mais, en droit congolais, il convient simplement de rappeler que depuis l'avènement du décret du 06 août 1950, le législateur avait déjà prévu des règles pénales spécifiques relatives à la délinquance des mineurs; et qu'il fallait dès cet instant commencer à soupçonner l'existence d'un droit pénal congolais des mineurs ou de l'enfance.

${ }^{21}$ J.L. De La Cuesta, "Le nouveau statut pénal du mineur en Espagne", RIDC, 1-2004, pp.159174. 
spécial, et d'autre part, un seuil supérieur, appelé aussi “ majorité pénale », qui est la limite au-delà duquel l'individu n'en a plus droit.

La fixation du second seuil n'a jamais posé beaucoup problème étant donné qu'elle se base sur le critère mécanique de l'âge et vacille généralement autour de la majorité civile, à savoir : entre 21,18 et 16 ans. C'est plutôt la fixation du premier seuil qui est plus complexe ${ }^{22}$ à cause principalement de la duplicité du critère de référence, en l'occurrence l'âge de l'enfant et/ou son discernement, ainsi que des enjeux qu'il porte, en frisant directement une irresponsabilité absolue.

En continuum, le Droit international des droits de l'homme, DIDH en sigle, a toujours préconisé un seuil d'âge en deçà duquel le mineur est présumé n'avoir pas la capacité d'enfreindre la loi pénale ${ }^{23}$, ou encore qu'il ne pourrait être suspecté, inculpé ${ }^{24}$, poursuivi ni convaincu d'une infraction et engager sa responsabilité pénale. Il a, toutefois, fallu attendre plusieurs années pour qu'il le fixe. C'est au Comité des droits de l'enfant $(\mathrm{CDE})^{25}$ que revient le mérite d'avoir proposé le seuil de 12 ans, plaidant en même temps pour son relèvement progressif à $14^{26}$ et puis 16 ans. Ce seuil, dans la lignée du droit romain, révèle l'importance d'exclure les plus jeunes enfants du nombre de ceux qui peuvent être pénalement responsables, d'une part, et compartimente le statut pénal des mineurs-délinquants en deux catégories en consacrant bel et bien la responsabilité pénale à l'égard de la frange du dessus du seuil d'âge fixé, d'autre part.

Il témoigne également le choix de ce droit (DIDH) pour le critère mécanique de l'âge qui lui apparaît plus rassurant (art. 5, 8 et 56 des Principes de Ryad) qu'un système qui laisse au juge ce pouvoir de le déterminer au cas par cas.

\footnotetext{
${ }^{22}$ J. Zermatten, “ L'observation générale $\mathrm{n}^{\circ} 10$ (2007) du Comité des droits de l'homme : les droits de l'enfant dans le domaine de la Justice des mineurs, Bulletin DEI, Juni 2007.

${ }^{23}$ Voir l'article 40 point 3 de la Convention Internationale des Droits de l'Enfant, du 20 novembre 1989. La présomption d'incapacité d'enfreindre la loi pénale se situe en amont. On considère en effet, que seul ce mineur est supposé être incapable de violer la norme pénale. En conséquence de ce qui précède, un tel mineur incapable en amont de violer la loi pénale, ne pourra pas non plus en aval, supporter les conséquences pénales de son acte en engageant pour cela sa responsabilité pénale.

${ }^{24}$ Article 4.1 Rés. 40/33, 13 et 14.c Rés.1997/30.

${ }^{25}$ Article 32-33 Obs. ${ }^{\circ}$ 10/2007, CDE ; Point 12 Rés. 18/12.

${ }^{26}$ L'Association internationale de droit pénal (AIDP) recommande aussi le seuil de 14 ans. Mais, l'âge médian international stagne à 12 ans. A ce jour, une quatre vingtaine d'Etats ont adopté un seuil inférieur à 12 ans parmi lesquels figurent principalement les pays de la common law mais aussi quelques pays romanistes à l'instar de la Suisse. Lire avec intérêt les Résolutions des Congrès de l'AIDP (1926-2004), ERES, n²0, 2009, Toulouse, 205-208, [en ligne] www.penal.org/sites/defaut/files/files/NEP\%2020\%20\%20français.pdf.
} 
$\mathrm{Vu}$ sous cet angle, le droit onusien se rapproche plutôt du modèle répressionnel.

Démarcation entre la reconnaissance de la responsabilité pénale et l'application de la sanction. Une autre particularité du droit pénal des mineurs tient de la démarcation qu'il convient de faire entre la reconnaissance de la responsabilité pénale et l'application de la sanction à l'agent. Il est évident que, lorsqu'ils sont reconnus responsables pénalement, les mineurs ne font pas généralement l'objet de la même tarification pénale que les majeurs. Quel que soit le système, ils n'encourent pas tous, les mêmes types de sanctions que certains textes peuvent nommer mesures. Ces dernières sont prononcées non seulement en fonction de la gravité des faits, mais aussi et surtout en tenant compte de l'âge et de la situation personnelle de l'enfant. Ainsi apparaît, particulièrement en droit interne, un autre seuil que l'on qualifiera de "seuil de sanctionnabilité ou de punissabilitê", lequel renvoie à l'âge à partir duquel un mineur peut encourir une mesure ou une sanction spécifique : un de ces seuils coïncide nécessairement avec le seuil inférieur à la responsabilité.

$\mathrm{Au}$ niveau interne des Etats, il conviendra d'évoquer à titre illustratif différentes législations en l'occurrence celles française, belge, allemande et congolaise.

En France ${ }^{27}$, par exemple, ces seuils sont fixés à 6/8 ans pour les mesures éducatives ; 10 ans pour les sanctions éducatives; et 13 ans pour la peine.

En Belgique, ces seuils sont différemment fixés comme suit notamment à 12 ans (seuil d'âge retenu par la Cour constitutionnelle belge). En effet, avant cet âge, le juge ne peut pas prendre de mesure à caractère pénal, mais uniquement des mesures de garde); et tout récemment dans certains $\operatorname{cas}^{28}$, à plus de 16 ans pour la peine.

\footnotetext{
${ }^{27}$ P. Bonfils et A. Gouttenoire, Droit des mineurs, Dalloz, $2^{\mathrm{ème}}$ éd., Paris, 2014, pp. 863-882;

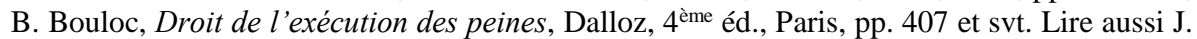
Pradel, Droit pénal comparé, Dalloz, 4ème éd., Paris, 2016, pp. 162-165.

${ }^{28}$ Il en est ainsi notamment de la mesure de dessaisissement permettant de soustraire à la juridiction des mineurs un jeune âgé de plus de 16 ans qui a commis un fait grave et de le juger comme un adulte (soit devant une juridiction des adultes soit devant une Chambre spéciale à trois juges, dont deux juges de la jeunesse, et qui reste attachée au Tribunal de la jeunesse, mais lui appliquera le droit pénal pour adultes). Voir les lois de 15 mai 2006 et 16 juin 2006 modifiant la loi du 8 avril 1965 relative à la protection de la jeunesse, le Code d'instruction criminelle, le Code pénal, le Code civil, la nouvelle loi communale et la loi du 24 avril 2003 réformant l'adoption.
} 
En Allemagne ${ }^{29}$, ce seuil est fixé à 14 ans tant pour des mesures qu'en ce qui concerne les peines.

Quant à la loi de protection de l'enfant en République démocratique du Congo, il convient de noter que le législateur congolais organise la démarcation de traitement en proscrivant l'application de certaines peines seulement et de certaines mesures aux mineurs à moins de 14 ans, l'enfant bénéficiera de la relaxe en matière pénale (art. $96 \mathrm{LPE}$ ); à moins de 15 ans, l'enfant ne pourra pas être placé dans un établissement de rééducation de l'Etat (art.117 al.2 LPE) ; à plus de 16 ans, le mineur ne peut pas être mis dans une institution publique à caractère social (art.113 al.2 LPE); et à moins de 18 ans, il ne peut être prononcé de peine de mort ni de servitude pénale à perpétuité pour des infractions commises par les enfants (art. 9, al. 2 LPE).

\section{La responsabilité pénale des mineurs en droit international humanitaire (DIH) : des Conventions de Genève au Statut de Rome.}

Les enfants sont les premières victimes des conflits armés, mais il n'est pas impossible qu'ils fassent partie de la cohorte des bourreaux ${ }^{30}$. Ils sont généralement enrôlés de force mais parfois aussi par nécessité ${ }^{31}$, et subissent des mauvais traitements de la part des recruteurs dans le but d'altérer leur capacité de discernement au moment de commettre le crime. Sauf que par moments, ils s'embrigadent volontairement, prennent goût à ce mode de vie et n'agissent toujours pas sous l'emprise de la drogue ou sous la contrainte.

\footnotetext{
${ }^{29}$ Dunkel Frieder, “Le droit pénal des mineurs en Allemagne : entre un système de protection et de justice", Déviance et Société, 2002/3, vol.26, pp. 297-313, [en ligne] http://www.cairn.info/revue-deviance-et-societe-2002-3-page-297.htm; "Le système judiciaire allemand", Ministère de la Justice, service des affaires européennes et internationales/Bureau du droit comparé, 21/02/2014, pp.6-8, en ligne sur [http://www.caangers.justice.fr/art_pix/1_1_1_fp_sj_allemagne.pdf, consulté le 23/03/2017.

${ }^{30}$ L'utilisation des enfants dans les forces armées est un phénomène ancien ; à la différence qu'aujourd'hui, à cause de la prolifération d'armes légères et du moindre coût de main d'œuvre qu'ils représentent, les enfants sont plus utilisés comme des combattants plutôt que des serviteurs, esclaves, espions, boucliers humains comme autrefois. Et, le phénomène se pérennise principalement en Afrique. Voir: Amnesty international, Attention: Enfantssoldats!, Dossier pédagogique 2012, pp.4-7, [en ligne] www.amnestyinternational.be/IMG/pdf/ dossierenfantssoldats-2.pdf ; Croix-Rouge de Belgique, Communauté francophone, Thématique: Enfants-soldats, [en ligne] http://www.croix-rouge.be/img/db/enfants-soldats-pdf ; O.H. Mouzayan, L'enfant soldat, Odile Jacob, Paris, 2003, pp. 9, 15-19; https://fr.wikipédia.org/wiki/enfant_soldat ; M. Tournier, "l'enfant soldat dans l'histoire", Bulletin No8, 01/2004, Amnesty Internationale/Section française, Commission enfants ; www.child-soldiers.org/francais.php

${ }^{31}$ Défendre leur pays, leur village, leur terre; venger la mort de leurs parents; salaire alléchant...
} 
Ces deux cas de figure, qui résument la situation des mineurs impliqués dans les conflits armés, ne peuvent continuer à être ignorés, ni confondus. Malheureusement, ils ne trouvent aucune solution claire dans la réglementation internationale: cette dernière préfère entretenir un flou poussant à imputer sur les recruteurs les violations graves commises par les mineurs engagés dans les hostilités, mais à garder silence ${ }^{32}$ sur les concernés eux-mêmes pendant qu'il se pose une véritable question de leur culpabilité, couplée à celle de la protection des droits des victimes.

\section{a. La non-exclusion d'une responsabilisation des mineurs pour crimes internationaux}

En ce qui concerne les mineurs, auteurs des crimes internationaux, les instruments internationaux préfèrent garder silence quant à la possibilité ou non de leur responsabilité pénale. Mais, ce silence ne semble être que de façade. Car, au-delà des débats d'école ${ }^{33}$, le constat est que le Droit international humanitaire n'interdit pas expressément toute idée de responsabilité pénale individuelle des mineurs pour crimes internationaux, quoiqu'il incite les systèmes nationaux à considérer les mineurs impliqués aux différents conflits armés comme des victimes et à privilégier leur démobilisation en vue de leur réinsertion via les modes alternatifs des règlements des conflits ${ }^{34}$. Le statut de prisonnier de guerre, que leur confèrent les protocoles additionnels de la Convention de Genève, ou les dispositions de l'article 40-2-a de la Convention Internationale des Droits de l'Enfant, CIDE en sigle, n'interdisent pas non plus leurs poursuites en cas de violation grave des lois et coutumes de guerre.

Assez souvent, la polémique émane principalement de la déclaration d'incompétence des juridictions pénales internationales à l'égard des personnes âgées de moins de 18 ans. Il en est ainsi de l'article 26 du statut de Rome de la Cour pénale internationale. C'est qu'assez facilement, on considère que cette disposition consacrerait une irresponsabilité pénale de ces

\footnotetext{
${ }^{32}$ H. Gibromont, "La prise en compte des enfants-soldats par les juridictions pénales internationales et internationalisés", Clinique de droit international pénal et humanitaire, 25/06/2014, URL : http://www.cdiph.ulaval.ca/blogue/la-prise-en-compte-des-enfantssoldats-par-les-juridictions-penales-internationales-et, du 16/09/2015; P. Manirakiza, " Les enfant face au système international de justice : à la recherche d'un modèle de justice pénale internationale pour les délinquants mineurs", L.J. QUEEN'S, 2009, p.722

${ }^{33}$ Pour avoir un aperçu sur les principaux courants doctrinaux sur cette question, lire l'excellent article de monsieur Luc akakpo ["Procureur c. X : les enseignements à tirer de la poursuite des enfants soldats pour crime contre l'humanité", $R G D$, (2012), (42), n¹, pp.9-56, disponible en ligne sur : http://id.erudit.org/iderudit/1026915ar].

${ }^{34}$ Point 3.6 des Principes de Paris, 16 et 21 des Principes du Cap.
} 
personnes. Et pourtant, une fois de plus, on ne peut déduire de cette disposition ni une responsabilisation ni une irresponsabilisation des mineurs. Car, les textes régissant les juridictions pénales internationales n'ont jamais, et rien ne les en empêchait, formellement consacré une irresponsabilité pénale au bénéfice des concernés, âgés de moins de dix-huit ans. Aussi, une " déclaration d'incompétence d'une juridiction exclut tout simplement la possibilité pour cette dernière de connaître de la cause, mais ne sous-entend nullement l'irresponsabilité de l'agent ${ }^{35} »$. Même dans l'hypothèse où cette juridiction serait la seule susceptible à connaître de tels faits, on évoquerait en réalité l'impunité pour absence de juridiction compétente et non l'irresponsabilité pénale.

Force est de constater que les juridictions pénales internationales n'ont pas de compétence exclusive. Elles ont souvent une compétence concurrente à celle des juridictions nationales ; même si il peut s'agir soit de la primauté, soit de la subsidiairité. On remarque en effet que ce sont les impératifs d'ordre économique ${ }^{36}$ qui sont souvent évoqués comme principales causes qui les obligent à se concentrer sur les " primo-délinquants ${ }^{37}$ et à laisser à leurs homologues nationales, la charge de juger les exécutants dont font partie généralement les mineurs. Il y a donc ici une sélectivité justifiée des justiciables. Les uns, plus importants à cause de leur forte influence dans la réalisation du crime, sont préférés par les juridictions pénales internationales ; tandis que les autres, moins importants que les précédents puisqu'étant intervenus au second plan, peuvent être jugés par les juridictions nationales compétentes.

\section{b. Seuil légal de l'enrôlement volontaire ou seuil de responsabilité pénale pour crimes internationaux?}

\footnotetext{
${ }^{35} \mathrm{~B}$. Wane bameme, La responsabilité pénale pour crime de guerre. Etude comparée des droits français et congolais, Thèse, AMU, (2012), p.235.

${ }^{36}$ L. Cote, " Justice pénale internationale : vers un resserrement des règles du jeu », RICR, 03/2006, n 861, pp. 51-63 ; Laucci C., “Quoi de “ spécial” au TSSL ?”, AYIL, vol.14, 2006, spéc. p.40, URL: lawlib. wlu.edu/CLJC/index.aspx?mainid=1067\&issuedate=2008 0321\&homepage $=$ no ; P. Xavier, Cours de Droit international pénal, M1, Faculté de droit et de science politique, AMU, [Inédit], 2010-2011.

${ }^{37}$ Ce terme ne doit pas être pris dans le sens généralement admis qui l'oppose à "récidiviste" et "délinquant d'habitude"; mais le sens utilisé par le professeur Xavier Phillipe [précité] comme étant les auteurs intellectuels.
} 
Il est aussi évoqué, comme cause de cette exclusion de compétence, le manque de consensus ${ }^{38}$, autour du seuil de responsabilité pénale qu'il a fallu éviter tout conflit entre les juridictions internationales et celles nationales. Ce qui confirme bien notre hypothèse selon laquelle l'idée de responsabilité a toujours été présente mais c'est plutôt la fixation de son seuil qui a posé problème. Or, lorsqu'on analyse minutieusement les règles du Droit international humanitaire, DIH en sigle, il semble se dégager un seuil [implicite] de quinze ans ${ }^{39}$. En effet, si l'interdiction de recrutement et celle relative à la participation des personnes âgées de moins de 18 ans dans les conflits armés ne sont plus discutables en Droit international des droits de l'homme (DIDH) et en Droit international humanitaire (DIH) ; seule la violation de la non-participation directe des personnes âgées de moins de 15 ans est assortie d'une sanction pénale.

Ne sont donc pas pénalement interdites d'une part, la participation indirecte des enfants de moins de quinze ans dans les conflits armés, et d'autre part, la participation directe de ceux de plus de quinze ans ${ }^{40}$. Malgré le lobbying des organisations transnationales et non-gouvernementales, l'incrimination consécutive à cette violation de la règle humanitaire n'a pas toujours été modifiée. Vide juridique ou seuil tacite de responsabilité, cet âge coïncide curieusement avec la pratique d'enrôlement volontaire des mineurs adoptée par beaucoup d'Etats ${ }^{41}$ qui sont parfois contraints, par la réalitét ${ }^{42}$, à envoyer

${ }^{38}$ R. Gachoud, “La guerre, un jeu d'enfants ? Enfants soldats ; la problématique des filles”, AYIL, précité.; Rapport du Comité préparatoire pour la création d'une cour criminelle internationale, AG/N.U, 53 ${ }^{\text {ème }}$ sess., Doc. N.U A/CONF183/2/Add 1 (1998), p.53 ; Rapport de la Représentante spéciale du Secrétaire général pour les enfants et les conflits armés, Doc. off. A.G. N.U, $65^{\text {ème }}$ sess., Doc. N.U. A/65/219 (2010), §.8, [En ligne], http://daccess-ddsny.un.org/doc/UNDOC/GEN/N10/474/22/PDF/N1047422.pdf?OpenElement

39 Il existe des statuts des juridictions internationales ou internationalisées qui sont allées en deçà du seuil de 15 ans mais dans la limite de 12 ans posée par le DIDH (art. 40-3-a CIDE, 3233 Obs. $n^{\circ} 10, \mathrm{CDE}$ ). La Chambre spéciale de la Cour d'Etat de Bosnie-Herzégovine l'a fixé à 14 ans ; alors que Tribunal spécial pour la Sierra Leone (article 7 du statut du TSSL) et le Panel de Timor l'avaient respectivement fixé à 15 et 12 ans.

${ }^{40}$ E. David, Principes de droit de conflits armés, Bruylant, 3 ème éd., Bruxelles, (2002), pp. $492-$ 493 ; E. David, F. Tulkens Et D. Vandermeersch, Code de droit international humanitaire, Bruylant, $4^{\mathrm{ème} e}$ éd., 2010, 932-934 ; M. Matheson Et D. Momtaz (Dir), Les règles et institutions du droit international humanitaire à l'épreuve des conflits armés récents, Académie de droit international de la Haye, (2010), p. 26.

${ }^{41} \mathrm{Si}$ au regard du protocole de 2000 de la CIDE, les mineurs ne peuvent donc pas combattre ni faire l'objet d'un enrôlement obligatoire dans les forces armées, ils peuvent néanmoins recevoir une éducation et une formation militaire dès le plus jeune âge. Et, ce même en Europe (Belgique, France, Royaume-Uni).

${ }^{42}$ Par exemple, plusieurs mineurs de 17 ans sont comptés parmi les soldats britanniques disparus au combat en 1982 (Malouines), en1991 (guerre du Golfe), en 1998/1999 (Mission internationale de maintien de la paix au Kosovo). 
ces derniers au combat même s'ils s'engagent généralement à ne pas le faire. Ainsi, malgré le refus de la Communauté internationale de se prononcer, de manière explicite, sur la question de la responsabilité pénale des mineurs en droit international, les mineurs d'au moins 15 ans peuvent engager leur responsabilité lorsqu'ils sont auteurs des crimes graves de droit international. Raison pour laquelle, l'on retrouve dans la jurisprudence interne et celle produite par des juridictions internationalisées quelques cas de poursuite.

\section{c. Cas de poursuite des mineurs devant les juridictions internationales}

Le cas Dominique ONGWEEN devant la Cour pénale internationale ${ }^{43}$ : une vraie fausse illustration. Depuis son arrestation, nombreuses sont des analyses abordant la question en s'en félicitant, mais surtout en insistant sur des poursuites effectives contre ce criminel de guerre. Il y en a même qui ont soutenu, qu'il s'agirait du premier cas de poursuite initiée par la Cour pénale internationale conte un mineur. Et pourtant, rien de tel ne saurait se justifier.

Il est certes vrai que cet individu s'est retrouvé dans les rangs des forces combattantes rebelles, dans son pays à l'âge mineur. Il est aussi vrai que plusieurs personnes soutiennent qu'il aurait, en ce moment-là, tout en étant mineur, commis des crimes graves. Il n'est pas moins vrai qu'aujourd'hui, aucun de ces faits n'est mis à sa charge devant la Cour pénale internationale. L'action judiciaire en mouvement devant cette institution ne se rapporte donc pas aux faits criminels qu'il aurait commis lorsqu'il était mineur. Il ne s'agit pas d'une action contre un mineur. Il ne s'agit pas non plus d'une action en rapport avec les faits commis par le suspect alors qu'il était mineur d'âge. Il s'agit en effet plutôt d'une action en justice, initiée contre l'actuel majeur de plus de 42 ans, qui, comme tout autre individu, avait été mineur, subi des actes infractionnels, et très probablement commis d'autres. Mais, l'individu est en effet plutôt traduit devant la Cour pénale internationale pour des faits commis à partir de sa majorité d'âge. Notre position semble confortée, étant donné que d'une part, né en 1975 et transféré au quartier pénitentiaire de la Cour pénale internationale le 21 janvier 2015 à 40 ans, la Procureur de la Cour a mis à sa charge 70 chefs d'accusation de crimes de guerre et de crimes contre l'humanité, qui auraient été commis entre le $1^{\text {ier }}$ juillet 2002 et le 31 décembre 2005, notamment "attaque contre une population civile, meurtre, viol, esclavage sexuel, torture, réduction en esclavage" ou encore "utilisation d'enfants de moins de 15 ans pour participer activement à des hostilités". Dès lors, seuls les faits commis à partir de son $27^{\text {ième }}$ année jusqu'à près de son 30 ième année d'âge qui sont pris en compte devant la Cour pénale internationale,

${ }^{43}$ Le Procureur c/Dominic Ongwen, ICC-02/04-01/15, in https://www.icc-cpi.int. 
et entrainant 4.107 victimes retenues par cette juridiction. D'autre part, au moment où s'est ouvert le procès à son encontre, les avocats de la défense ont choisi d'arguer les traumatismes subis par leur client lorsqu'il était mineur d'âge, étant donné qu'il a été kidnappé lui-même entre 10 et 13 ans, mais sans accepter de soumettre leur client à une consultation d'expert. Cette ligne de défense oriente vers la possibilité pour lui de plaider non coupable en se fondant éventuellement sur une cause subjective d'irresponsabilité pénale (article $31 \mathrm{du}$ statut de la CPI), en l'occurrence l'altération de son discernement au moment des faits, à défaut de son abolition, et même la contrainte irrésistible.

La Cour pénale internationale, étant incompétente à juger les personnes qui, au moment des faits étaient âgées de moins de dix-huit ans ${ }^{44}$, ne peut en aucune manière, et sous aucune justification fondée, se déclarer compétente à juger une personne pour des faits commis alors qu'elle était âgée de moins de dix-huit ans.

Mais, c'est le Tribunal spécial pour la Sierra-Léone, l'illustration d'une juridiction pénale internationalisée ${ }^{45}$, qui a été la première à avoir, non seulement doté le droit international d'une jurisprudence en matière de conscription et d'utilisation d'enfants soldats constituant le crime de guerre, mais aussi envisagé en vertu de son statut ${ }^{46}$ de poursuivre les mineurs âgés de

\footnotetext{
${ }^{44}$ L'article 26 du statut de Rome de la Cour Pénale Internationale du 17 juillet 1998, ratifié par la République démocratique du Congo le 30 mars 2002, entré en vigueur le $1^{\text {ier }}$ juillet 2002, traitant de l'incompétence à l'égard des personnes de moins de 18 ans, prévoit ce qui suit : " $L a$ Cour n'a pas compétence à l'égard d'une personne qui était âgée de moins de 18 ans au moment de la commission prétendue d'un crime".

${ }^{45}$ P. Pazartzsis, “Tribunaux pénaux internationalisés : Une nouvelle approche de la justice pénale (inter)nationale", $A F D I, 49$ (1), (2003), pp. 641-661, spéc.646.

${ }^{46} \mathrm{~L}$ 'article $7 \mathrm{du}$ statut du Tribunal spécial pour la Sierra Leone, issu de l'accord entre les Nations Unies et le Gouvernement de la Sierra Leone, conformément à la résolution du Conseil de sécurité 1315 (2000) du 14 août 2000, traitant de la compétence de cette juridiction sur les êtres humains âgés de 15 ans, prévoit à peu près ce qui est traduit ci-contre : " 1 . Le Tribunal spécial n'a pas compétence sur toute personne qui était sous l'âge de 15 ans au moment de la commission prétendue d'un crime. Si une personne qui était au moment de la prétendue commission du crime entre 15 et 18 ans, devant la Cour, il ou elle doit être traité avec dignité et un sens de valeur, en tenant compte de son jeune âge et de l'opportunité de promouvoir sa réhabilitation, la réinsertion dans l'hypothèse d'un rôle constructif dans la société, et conformément aux normes internationales des droits humains, en particulier les droits de la l'enfant. 2. Dans la disposition d'une affaire contre un mineur délinquant, le Tribunal spécial visera dans tous les cas : l'orientation des soins et des ordonnances de surveillance, des ordonnances de service communautaire, le conseil, le placement familial, programmes de formation correctionnelle, éducative et professionnelle, des écoles agréées et, le cas échéant, des programmes de désarmement, de démobilisation et de réinsertion ou de programmes de protection de l'enfance".
} 
quinze ans au moment des faits pour les crimes de droit international humanitaire relevant de sa compétence ratione materiae.

Cette question s'est inéluctablement imposée à lui, étant donné que le conflit armé sierra-léonais (1991-2001) a été essentiellement alimenté par des jeunes, tentant d'échapper à la pauvreté ou tenus par l'idée de survie après avoir été enrôlés de force. Des auteurs soutiennent même que "la communauté internationale était généralement mal à l'aise [face] à cette idée [...] mais, en raison de leur nombre et de leur implication, plusieurs groupes sierra-léonais, y compris les chefs spirituels de diverses confessions, estimèrent qu'il convenait de les poursuivre" ${ }^{47}$. C'est ainsi que ce Tribunal a dû fonder ses poursuites sur le critère de l'opportunité (nécessité) déduit de la gravité et de l'ampleur des crimes commis.

Deux procédés ont été mis en place : la voix judiciaire via le Tribunal spécial pour la Sierra-Léone, TSSL en sigle, et la voie extra-judiciaire via la Commission vérité et réconciliation, CVR en sigle.

Le Procureur David Crane, qui a jugé inopportun d'utiliser la voie judiciaire ${ }^{48}$, a transféré des nombreux mineurs à la Commission vérité et réconciliation, CVR en sigle, qui n'avait pas pour mission d'établir leur responsabilité pénale mais plutôt d'analyser les motivations derrière leur comportement dans une optique de réconciliation, de pacification sociale, de prévention et de devoir de mémoire. Ce processus finissait souvent par la reconnaissance par les auteurs de leur culpabilité et la demande du pardon. Le Tribunal spécial pour la Sierra-Léone, TSSL en sigle, a donc, constitué l'exemple où la vulnérabilité des enfants ainsi que leur responsabilité ont été conjointement prises en considération de façon exhaustive suivant des mécanismes non-judiciaires.

\footnotetext{
${ }^{47}$ T. Perriello Et M. Wierda, “Le Tribunal spécial pour la Sierra-léone sur la sellette », ICTJ, 05/03/2005, p.14, URL : https://www.ictj.org/sites/default/files/ICTJ-SierraLeone-TribunauxHybrides-2006-French.pdf du 11/02/2016. Voir aussi E. Blunt, "Paix fragile en Sierra Léone », Le Monde Diplomatique, 12/1999, p. 14 ; Galy M., "Libéria, une guerre oubliée », Le Monde Diplomatique, 09/1994, p.11 ; Un autre témoignage d'un mineur ayant combattu en RD Congo, rapporté par l'ONG Vision du Monde, confirme cette perception [www.visiondumonde.fr/news/temoignage-enfant-soldat consulté le 10/03/2016].

${ }^{48}$ D. Crane, "Strike Terror NO More : Prosecuting the Use of Children in Times of Conflict The West African Extreme », International Criminal Accountability, K. Arts Et V. Popvski (Dir.), Hague Academic Press, Pays-Bas, 2006 ; M. Maystre Et A. Werner, " Un modèle de tribunal " internationalisé » : analyse du et perspectives sur le TSSL », Droit international pénal, R. Kolb (Dir.), Bruylant, Bruxelles, 2008, P. 403 ; T. Perriello Et M. Wierda, article précité.
} 
Un autre cas à évoquer est celui revenu aux Panels spéciaux ${ }^{49}$ du Timor Leste devant lesquels a été poursuivi, sous le chef d'accusation de crime contre l'humanité, d'extermination ou de tentative d'extermination et d'autres actes inhumains, un mineur âgé de quatorze ans au moment des faits qui avait tué trois personnes à la machette dans le cadre d'une tuerie généralisée.

Mais, alors qu'il y avait forte probabilité d'aboutir à sa condamnation, il y a eu une entente avec la défense, et la juridiction a retenu en effet plutôt le meurtre non prémédité (punissable en droit pénal indonésien) dont il fut reconnu coupable le 28 octobre $2002^{50}$. Il s'agit là de la première juridiction pénale internationale, quoiqu'hybride, ayant inculpé un mineur dans le cadre d'une procédure pénale ${ }^{51}$.

Cette décision, rarement commentée en doctrine, est souvent critiquée au motif que plusieurs garanties consacrées par le droit international en matière de justice pour enfants n'ont pas été respectées. Mais, il sied de souligner à ce sujet que, d'une part, l'organe d'accusation de cette juridiction était totalement internationalisé tandis que son siège l'était partiellement, et d'autre part, les règles de fonctionnement de cette juridiction intégraient tous les principes du droit pénal des mineurs consacrés en Droit international des droits de l'homme, DIDH en sigle, en Droit international humanitaire, DIH en sigle et en droits internes des Etas modernes, à savoir : un seuil de responsabilité pénale à 12 ans (supérieure à certains Etats), le caractère légal et nécessaire de la détention du mineur et la brièveté de toute peine d'emprisonnement. Dans ces conditions, il convient de s'interroger, en vue de la confirmation de la thèse soutenue, afin de savoir comment une juridiction ainsi régie et composée, est-elle parvenue à admettre l'hypothèse d'une poursuite pénale à l'encontre d'un mineur de 14 ans pour des faits constitutifs de crimes internationaux si une telle approche était contraire au droit international.

Néanmoins, il faut reconnaître que, en dehors des cas précités, et peut-être celui du jeune Omar KHADR ${ }^{52}$, les exemples sont à chercher. La politique

49 Appelés aussi "Chambres spéciales pour les crimes graves de Timor".

50 DILI District Court, Special Panel for Serious Crime, Prosecutor c/ X, n ${ }^{\circ}$ 04/2002, $12 / 12 / 2002$, [en ligne] https://www.wcl.american.edu/warcrimes/wcro_docs/collections/spscet/SPSC, East, Timor, Judgmts, Indmts \& Docs/X/, consulté le 12/02/2016

${ }^{51}$ L. Akakpo, Op. Cit.

${ }^{52}$ Sans entrer dans les spécificités du droit pénal canadien, du droit international et du droit pénal américain, il s'agit, dans ce cas d'espèce, d'un jeune canadien, âgé de 14 ans au moment des faits, qui a été condamné par une commission militaire américaine en date du 25 octobre 2010, pour crimes de guerre, de complot, de soutien matériel au terrorisme, d'espionnage et de meurtre. 
pénale prônée par le droit international reste, d'une part, la fixation des limites à ne pas franchir à une justice en continuel alignement sur le modèle des adultes, et d'autre part, le référencement du mineur, quelle que soit l'infraction commise, devant son juge national naturel compétent.

Il en est ainsi également du droit congolais, qu'il convient d'analyser.

\section{De La (L'ir) Responsabilite Penale Des Mineurs En Droit Positif Congolais : Une infirmation dogmatique}

Etant donné que la République démocratique du Congo a ratifié la quasitotalité des traités évoqués précédemment, il importe de souligner que ces instruments juridiques doivent régulièrement et normalement s'y appliquer.

Indépendamment, mais parfois aussi à la suite de ces traités, le droit interne congolais prévoit un corpus de règles sur la question relative à la responsabilité pénale des mineurs. Cependant, tout porte à croire que le débat autour de la question a été orientée, puisque conduite par certaines motivations non clairement élucidées. La doctrine dominante, ayant été construite à l'époque dans la moule protectionnelle du droit belge, a retenu et fait comprendre plusieurs générations de juristes une certaine irresponsabilité pénale totale des personnes âgées de moins de dix-huit ans, sans pour autant qu'une telle affirmation ne trouve justification textuelle. C'est qu'à cette époque, aucun texte congolais régissant la matière ne l'affirmait de manière expresse. Durant toute cette période, l'histoire ne renseigne pas l'existence d'un autre courant doctrinal à l'affrontement duquel jaillirait, si pas la vérité, mais peut-être un autre son de cloche pouvant conduire à la relativisation de certaines positions d'écoles en matière pénale, manifestement dogmatiques mais véritablement pourtant non textuelles.

Pour mieux s'en rendre compte, il convient et il suffit de scruter les deux textes qui ont organisé la gestion de la délinquance des mineurs en droit congolais, savoir: le décret du 06 décembre 1950 sur l'enfance délinquante et la loi $\mathrm{n}^{\circ} 09 / 001$ du 10 janvier 2009 portant protection de l'enfant.

\section{a. La responsabilité pénale des mineurs sous le décret du 06 août 1950 : Une responsabilité dogmatiquement infirmée}

Sans être le premier texte de droit à caractère pénal à s'intéresser au mineur au Congo ${ }^{53}$, le décret du 06 décembre 1950 a eu le mérite d'avoir rassemblé

53 Il y a lieu de citer ces différents autres textes qui l'ont précédé :

le Décret du 04 mai 1885 sur la correction paternelle ;

l'Arrêté du 05 mai 1896 sur le vagabondage et la mendicité ; 
toutes les règles éparses du droit colonial en la matière, en les mettant sous le contrôle d'un juge spécial. On retiendra que ce texte fut, à quelques exceptions près, essentiellement inspiré par la loi belge de 1912. Il consacre globalement les principes fondamentaux du droit des mineurs et même du droit pénal des mineurs, savoir : la minorité d'âge ${ }^{54}$, la spécificité juridictionnelle ainsi que la spécificité sanctionnelle ${ }^{55}$. A ces principes, la touche protectionniste ajoutera le contrôle socio-judiciaire des mineurs affichant des comportements déviants et l'absence de seuil de responsabilité pénale dans le décret de $1950^{56}$.

\section{a) Une irresponsabilité dogmatique et conjecturale}

Le droit congolais, à la suite du droit belge qui l'avait inspiré en 1950, choisit de remplacer la peine par les mesures (art. 5, al. 2 D). C'est justement cette déqualification qui, à y regarder de près, n'a d'ailleurs concerné que la peine ${ }^{57}$, est évoquée à l'appui de la thèse d'irresponsabilité pénale des mineurs. Et pourtant, le législateur congolais prévoit simplement ce qui suit d'abord à l'article 5 du Décret:" Si un mineur a commis une infraction, le seul juge compétent pour en connaître au premier degré sera le juge de [paix] siégeant avec officier du ministère public, magistrat de carrière. La peine sera remplacée par une mesure de garde, d'éducation ou de préservation d'après les distinctions suivantes »; ensuite à l'article 6 du Décret : " Quelle que soit la qualification pénale du fait commis, le juge pourra, selon les circonstances, soit réprimander l'enfant et le rendre aux personnes qui en avaient la garde, avec injonction de mieux le surveiller à l'avenir, soit le confier jusqu'à sa vingt et unième année à une personne, à une société ou à une institution de charité ou d'enseignement, publique ou privée, soit encore le mettre jusqu'à sa vingt et unième année à la disposition du gouvernement".

- le Décret du 23 mai 1896 donnant également qualité au magistrat pour intervenir en cas de vagabondage ;

- l'Arrêté du 03 janvier 1911, l'Ordonnance 76/J du 15 octobre 1931 sur le régime pénitentiaire...etc.

${ }^{54}$ La minorité d'âge a été arrêtée à 18 ans à l'article premier du décret du 06 décembre 1950 sur la délinquance juvénile, puis à 16 ans dans le même article $1^{\text {er }}$ du même décret, lorsqu'il a été modifié et complété par l’Ordonnance-Loi nº78-016 du 4 juillet 1978.

${ }^{55}$ Les articles 5 et 6 du décret du 06 décembre 1950.

${ }^{56}$ La majorité pénale sera fixée à 18 ans révolus et intégrée dans le Code pénal à l'article 20 ter du décret du 30 janvier 1940 portant Code pénal, modifié et complété par la loi n ${ }^{\circ} 15 / 022$ du 31 décembre 2015 entrée en vigueur trente jours après sa publication au journal officiel de la République démocratique du Congo, du 29 février 2016.

${ }^{57}$ Contrairement à une certaine opinion, aucun article et même pas l'article 6 du décret du 06 décembre 1950 n'a consacré une substitution du terme "infraction" par "manquement", comme la loi belge de 1912. Le législateur congolais n'évoque l'expression " manquement qualifié d'infraction par la loi pénale" qu'uniquement dans la loi sur la protection de l'enfant, LPE en sigle (notamment à l'article 2, point 9) en définissant l'enfant en conflit avec la loi'. 
En effet, après avoir affirmé la possibilité pour un mineur de commettre une infraction, le législateur a simplement obligé le juge à remplacer la peine par différentes mesures. Mais, déduire d'une telle spécificité sanctionnelle, une quelconque irresponsabilité pénale de tout mineur, comme longtemps soutenu par le passé, n'est que dogmatique, et même conjectural puisqu'il s'agit là d'une opinion fondée sur des simples apparences. Le décret du 06 août 1950 relatif à l'enfance délinquante ne comportait pas moins de disposition à coloration ou à connotation pénale ${ }^{58}$.

Lorsqu'on analyse le régime sous la doctrine positiviste, force sera de constater que la minorité ait toujours fait l'objet d'une réaction sociale particulière et différente des autres causes subjectives d'irresponsabilité pénale.

D'ailleurs, la bonne analyse démontre aujourd'hui que cette irresponsabilité pénale des mineurs n'est consacrée nulle part dans un texte qui, bien au contraire, établit " un recours systématique à une procédure judiciaire pour

\footnotetext{
${ }^{58}$ Il importe de souligner que différents articles du décret du 06 décembre 1950 relatifs à l'enfance délinquante étaient très évocateurs sur la question. En effet, différentes notions de droit pénal pouvaient y être retenues : D'abord, avec la révision intervenue à travers l'Ordonnance-Loi n ${ }^{\circ} 78-016$ du 4 juillet 1978, il fallait entendre par mineur, au sens de l'article premier dudit décret, l'enfant âgé de moins de seize ans accomplis au moment du fait. De sorte que si tout mineur était, comme enseigné à l'époque, irresponsable pénalement, il fallait normalement retenir que la personne âgée de plus de seize ans pouvait engager sa responsabilité pénale et subir toute peine (y compris celle de mort et de servitude pénale à perpétuité). Ensuite, les mesures portées par l'article 2 de ce Décret ne pouvaient principalement être prises qu'en cas de mendicité ou de vagabondage. Ce qui veut dire que l'article 2 ne concernait pas d'autres comportements en dehors de ceux clairement indiqués. En plus, sans consacrer l'irresponsabilité pénale, le second alinéa de l'article 5 remplace simplement la peine par une mesure de garde, d'éducation ou de préservation d'après les distinctions suivantes. On retiendrait ici la spécificité sanctionnelle. Enfin, on compte quatre articles qui prévoyaient la mesure de mise à la disposition du gouvernement (article 6 du décret du 6 août 1950, et pourtant le décret du 30 janvier 1940 portant Code pénal la prévoit aussi parmi les peines portées par l'article 5 point 8 ainsi qu'aux articles $14 \mathrm{~d}$ à $14 \mathrm{k}$ ), avec possibilités de sa prolongation, ne manquant pas de connotation pénale. Il en est ainsi de la possibilité de prolongation d'abord audelà de la vingt et unième année de l'enfant pour un terme qui ne pourra dépasser sa vingtcinquième année, en cas d'une infraction punissable de plus de cinq ans de servitude pénale mais non punissable de la peine de mort ou de la servitude pénale à perpétuité (article 7) ; ensuite, au-delà de la vingt et unième année de l'enfant pour un terme de vingt ans au maximum, en cas d'une infraction punissable de la peine de mort ou de la servitude pénale à perpétuité (article 8) ; enfin, en cas de perversité morale trop caractérisée, l'enfant devait être interné dans un établissement de rééducation de l'Etat pour 2 à 10 ans, et même pour ce qui est de l'infraction punissable de mort ou de servitude pénale à perpétuité, au-delà de la vingt et unième année de l'enfant. Il y a lieu d'ajouter aussi l'article 17 du décret du 6 août 1950 qui prévoyait qu'en cas d'un mineur vicieux, ou si aucun particulier ni institution ne pouvait l'accueillir, il pouvait être gardé préventivement dans une prison pour une durée ne dépassant pas deux mois, bien évidemment sous un régime spécial.
} 
tous les mineurs infracteurs» ${ }^{59}$ et déviants ; reconnaît et donc n'écarte pas la possibilité pour l'enfant de commettre une infraction, contrairement à la Convention internationale des droits de l'enfant, CIDE en sigle, laquelle prône la fixation d'un seuil d'âge en dessous duquel l'enfant est présumé n'avoir pas la capacité d'enfreindre la loi pénale ${ }^{60}$.

En plus, en tant que norme pénale et dérogatoire, l'irresponsabilité pénale de tous les mineurs aurait dû être consacrée assez clairement. Bien plus, entant que cause subjective d'irresponsabilité pénale, cette minorité est soumise à une double exigence de textualité ou de normativité, puisque d'un côté le texte pénal général devrait le consacrer, et que de l'autre côté celui spécial aurait dû le confirmer.

\section{b) Une déduction de l'irresponsabilité pénale à partir de l'attribution de compétence à une juridiction spécialisée}

Par moments, on se permet de déduire l'irresponsabilité pénale des mineurs, à partir de l'attribution de la compétence exclusive de la délinquance juvénile à un juge spécialisé. Mais une telle idée, plutôt que d'être convaincante, n'est en réalité que déroutante. Puisqu'à y regarder de près, la même juridiction instituée, à cet effet, n'était autre qu'une juridiction de droit commun (juge de première instance, devenu bien après, juge de paix) statuant au premier degré, dans une chambre pénale spécifique. Ce qui signifie que l'affaire intégrait en appel le circuit pénal ordinaire étant donné qu'aucune chambre spécifique équivalente n'était prévue au niveau du Tribunal de grande instance, même pas en Cassation.

Par ailleurs, doit-on encore, à l'ère de la profusion et de la spécialisation juridictionnelles que connaît le droit moderne, déduire l'irresponsabilité pénale simplement à partir de l'attribution d'une compétence à un juge spécialisé ? Une réponse affirmative à cette question conduirait à déduire

\footnotetext{
59 “ La protection légale et judiciaire des enfants en RDC : Problèmes centraux et propositions", Document de discussion, BICE - MONUC/Section Protection de l'Enfant - Save the Children/UK - UNICEF, Kinshasa, Déc 2004, p.8 [§. Les enfants criminellement responsables]. Voir aussi "Recueil sur la minorité: Analyse et commentaires de la législation applicable aux mineurs en RDC", BICE, Kinshasa, (2002), pp. 21-22, [en ligne] http://www.kirainternational.org/downloads/recueilminoriterdc.pdf, consulté le 26/02/2015.

${ }^{60}$ L'article 40, point 3 de la Convention Internationale des Droits de l'Enfant du 20 novembre 1989 prévoit à cet effet ce qui suit : “ 3. Les Etats parties s'efforcent de promouvoir l'adoption de lois, de procédures, la mise en place d'autorités et d'institutions spécialement conçues pour les enfants suspectés, accusés ou convaincus d'infraction à la loi pénale, et en particulier : D'établir un âge minimum au-dessous duquel les enfants seront présumés n'avoir pas la capacité d'enfreindre la loi pénale ; De prendre des mesures, chaque fois que cela est possible et souhaitable, pour traiter ces enfants sans recourir à la procédure judiciaire, étant cependant entendu que les droits de l'homme et les garanties légales doivent être pleinement respectés".
} 
également l'irresponsabilité pénale des militaires dont les infractions purement militaires relèvent de la compétence d'un juge spécialisé ${ }^{61}$.

\section{c) Une remise en question des archétypes en droit comparé}

Il arrive également que l'on tente de trouver justification de cette prétendue irresponsabilité pénale de l'enfant en recourant au droit comparé, et particulièrement au droit belge. Alors qu'en réalité, l'analyse comparée révèle que depuis les temps immémoriaux, le mineur nage toujours entre une irresponsabilité absolue et une responsabilité atténuée ${ }^{62}$. La responsabilité pénale étant, à l'origine objective, la minorité était perçue comme une inaptitude sanctionnelle ${ }^{63}$. L'ère classique lui a donné un fondement théorique et subjectif sans pour autant la libérer de l'emprise sanctionnelle préclassique. Les aspects criminologiques n'ont été pris en considération qu'à l'apothéose de l'Etat-providence : le mineur-délinquant, perçu comme une victime de l'inadaptation sociale et du défaut de la surveillance parentale, sera alors soumis à une pénologie particulière, à vocation préventive et éducative.

Dès lors, apparaissent deux idées sur la fonction de la responsabilité des mineurs, une responsabilité "des causes ${ }^{64}$ et une responsabilité des " résultats", s'opposeront et induiront deux grands modèles de droit pénal des mineurs. D'un côté, le modèle dit "répressionnel"65, dit également "pénal", de "justice" ou "pénitentiaire", lequel consacre clairement la responsabilité pénale des mineurs d'après le procédé classique (causalité, culpabilité, imputabilité) et applique aux mineurs une tarification pénale soit minimale, soit identique aux majeurs (USA, Angleterre, Pays de Galle). De l'autre côté, le modèle dit " protectionnel" simplement de "protection", qui estime que les mineurs sont des victimes (de leur condition de vie) qu'il sied de leur appliquer des mesures de protection et

\footnotetext{
${ }^{61}$ Article 76 de la Loi n ${ }^{\circ}$ 023/2002 du 18 novembre 2002 portant Code judiciaire militaire. Ce raisonnement peut aussi être appliqué aux tribunaux de commerce qui connaissent toutes les infractions relatives à la législation économique et commerciale (article 17, alinéa 2 de la Loi $\mathrm{n}^{\circ} 002 / 2001 \mathrm{du} 03$ juillet 2001).

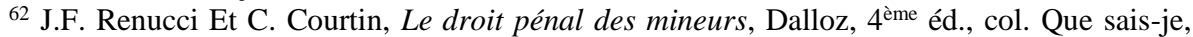
Paris, 2001, p.59. Voir aussi J. Bart, Histoire du droit privé de la chute de l'Empire romain au XIX ${ }^{\text {ème }}$ siècle, Montchrestien, 1998, pp. 67-68 ; C. Blatier, La délinquance des mineurs :

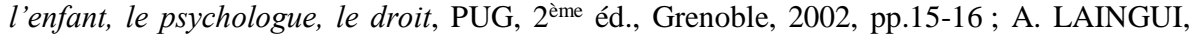
Petite histoire illustrée du droit pénal, Litec, Paris, (2010), pp.113-114.

${ }^{63}$ Le droit pénal préclassique est construit autour de l'acte (fait) criminel qu'il fallait bannir par la neutralisation du délinquant. A cet effet, le corps du mineur apparaissait comme ne pouvant pas supporter le même supplice que celui d'un adulte, d'où l'admission de l'adoucissement de la peine à son égard.

64 J. Chazal, Etudes de criminologie juvénile, Paris: PUF, (1952), pp. 11-13.

${ }^{65}$ Dit aussi " pénal", " de justice" ou " pénitentiaire".

${ }^{66}$ Dit aussi " tutélaire", " paternaliste" ou " de protection".
} 
d'éducation (Belgique, Canada, Portugal). Ce modèle adopte souvent " des modalités d'évitement du procès pénal avec des procédures déjudiciarisées"67 et accorde des larges pouvoirs d'appréciation aux juges d'enfants et implique d'autres organes, essentiellement non judiciaires, au traitement de l'enfant mis en cause. S'il faut cartographier, on constatera à cet effet, qu'il apparaît une cartographie binaire qui distingue les Etats anglo-saxons d'obédience ultralibérale, des Etats romanistes d'idéologie socialiste. Ce qui amène certains à considérer que le système protectionnel est inspiré de la doctrine "parens patrice", tandis que le modèle répressionnel, de la " due process of law", soucieuse des garanties procédurales ${ }^{68}$.

Mais, en réalité aucune classification n'est aujourd'hui rigoureusement autonome et satisfaisante. Aucun critère n'est réellement pertinent. Aucun système ne reste figé et recroquevillé à lui-même; bref, aucun modèle ne s'auto-suffit. Si l'on ne considère que le critère de la peine ${ }^{69}$, il s'avère que toutes les mesures, aux vertus protectrices, appliquées aux mineurs ne sont pas toujours exemptées des attributs de la peine ${ }^{70}$ (Allemagne, Belgique, France). La Suisse par exemple, qui n'applique que des mesures éducatives à l'égard des mineurs délinquants, consacre un seuil inférieur de la responsabilité pénale, qui d'ailleurs a toujours été l'un des plus bas sur le continent d'Europe (7 ans avant 2007 et 10 ans après 2007). De même, certains systèmes, qui se revendiquent tutélaires ou qui ont consacré expressis verbis l'irresponsabilité pénale du mineur admettent pourtant la possibilité d'appliquer à certains mineurs le droit pénal commun (Allemagne, Belgique, Canada). Bien plus, tous organisent une action juridique visant à la fois la victime (obtention de la réparation) et le mineur-délinquant (payer sa dette envers la société) : la nature d'une telle action, vu toutes ses imbrications, pourrait bien être complexe mais dépasse manifestement le cadre de l'action civile. Ce qui rapproche le modèle paternaliste, pourtant présenté comme plus tendre et plus évolué, de la conception objective ${ }^{71}$ de la responsabilité pénale. C'est ainsi que certains

\footnotetext{
${ }^{67}$ P. Bonfils, “ Chronique de droit pénal des mineurs », RIDP, 2009/1 Vol. 80, pp. 307-315.

68 O. D'amours, " Les grands systèmes. Modèle de protection, modèle de justice et les perspectives d'avenir », Service social, v.47/n³-4, 1998, pp. 15-40, consulté le 11/11/2013. URL : http://id.erudit.org/iderudit /706794ar

69 "Peine" = Responsabilité pénale = répressionnel ; "Mesure de sûreté" = irresponsabilité pénale $=$ protectionnel.

${ }^{70}$ Arrêt Boumar C/ Belgique, CEDH, n 9106/80, 29 février 1988 ; Willems L., " Un point de vue critique des carences de l'application de la loi du 08 avril 1965", Déviance et société, 9 (2), (1985), pp.151-157, spéc. p.152.

${ }^{71}$ J. Pradel Et A. Varinard, Les grands arrêts du droit pénal général, Dalloz, 9 e éd., Paris, 2015, pp. 667-671.
} 
qualifient cette subversion langagière d'hypocrisie juridique ou de rhétorique de déguisement ${ }^{72}$.

Au final, l'évolution du droit pénal des mineurs démontre que derrière toute idée de protection des mineurs se cache toujours et déjà un modèle pénal en quête d'efficacité ${ }^{73}$. Puis, dans tout système sanctionnel sont pris en compte les acquis et connaissances scientifiques de la défense sociale qui n'ont jamais été totalement remis en cause. Par pragmatisme, moult systèmes juridiques renoncent à ce carcan archétypique et idéologique et se mettent à la recherche d'une troisième voie ${ }^{74}$. Il s'agit de la voie de l'équilibre, de la réalité et de la mixité à travers l'hybridation des deux systèmes existants. D'où la tendance générale, même parmi les plus protectionnistes comme le Canada ${ }^{75}$, vers la répression, la responsabilisation des mineurs ${ }^{76}$ et la justice restaurative.

Et, si l'on ne s'en tenait qu'à la Belgique, laboratoire de la défense sociale, les travaux parlementaires de la loi du 05 mai 1912, ayant instauré le modèle protectionnel, laissent entrevoir " avant tout un réflexe défensif, un souci de gestion rationnelle et efficace d'une délinquance en accroissement chronique plutôt qu'une indulgence teintée d'idéalisme humaniste" "77. Le régime proposé devrait comporter " [...] dans de nombreux cas une plus grande

72 Y. Cartuyvels, "Les grandes étapes de la justice des mineurs en Belgique, continuité, circularité ou ruptures?", Journal du droit des jeunes, (207), (2001), pp.13-33; P. Cornelis, "Présentation des travaux de la commission nationale belge pour la réforme de la loi sur la Protection de la Jeunesse", In "Des réponses claires face à la délinquance des mineures", Actes de colloque organisé à Bruxelles par le MR en 2005, URL: www.mrchambre.be/.../delinquance\%20juveni...

${ }^{73} \mathrm{~T}$. Moreau, "La prise en charge des mineurs difficiles est-elle considérée comme une priorité dans notre société ?", In "Des réponses claires face à la délinquance des mineures", Op. Cit.;

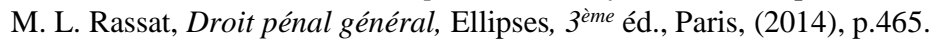

${ }^{74}$ C. Maes, "La justice juvénile dans le monde, ses systèmes, ses objectifs : les modèles", Séminaire de formation en justice des mineurs pour magistrats et autres acteurs en justice juvénile de l'Afrique francophone, Ouagadougou 2004, IDE, Working Report, 1-2005, pp.1732 ; J. Zermatten, "La loi fédérale régissant la condition pénale des mineurs", IDE, Working Report, 3-2004, pp. 9-15.

75 J. Trépanier, "L'avenir des pratiques dans un nouveau cadre légal visant les jeunes contrevenants", 2003-04, º34, RDUS, pp.47-88, spéc.51.

${ }^{76}$ F. Bailleau Et Y. Cartuyvels (Dir), La Justice pénale des mineurs en Europe, Déviance et Société, 2002/3, vol.26 ; F. Bailleau et al., "La criminalisation des mineurs et le jeu des sanctions", Déviance et Société, 3 (33), (2009), pp. 255-269.

77 "Analyse des législations en matière de justice des mineurs : Cas de la Belgique", Ministère de la Justice française/Service des affaires européennes et internationales, CNRS-JURISCOPE, Paris, 10/04/2009, p.4 ; D. De Fraene, "Historique de la réponse donnée par le législateur au problème de la délinquance juvénile", In "Des réponses claires face à la délinquance des mineurs", Op.Cit. 
sévérité que le régime du Code pénal"78. Bien que prônant la théorie d" irresponsabilité pénale des mineurs ${ }^{79}$ ", le législateur de 1912 n'a jamais eu le courage de l'affirmer dans le corps du texte et le régime qu'il avait mis en place n'a jamais prétendu vouloir extirper le mineur-délinquant du droit pénal, ou de sa responsabilité pénale, mais avait plutôt pour ambition de contourner les règles pénales classiques qui empêchaient toute intervention en amont de la commission de l'infraction. "Même si on admettait une irresponsabilité pénale, elle devrait être relativisée et réduite à la peine [...]. Le système ne tarda pas à montrer ses limites qu'une réforme, appelée de tous les vœux, viendra combler en 1965 qui, malgré les multiples modifications qu'elle connaîtra, n'aura non plus le courage de consacrer l'irresponsabilité pénale des mineurs" ${ }^{\prime 80}$. Cette réforme de 1965 se bornera à instituer une présomption de non discernement. L'article 38 de cette loi belge du 8 avril 1965 relative à la protection de la jeunesse décide donc que si le tribunal de la jeunesse estime inadéquate une mesure de garde, de préservation ou d'éducation, il peut par décision motivée se dessaisir et renvoyer l'affaire au ministère public aux fins de poursuite devant la juridiction compétente en vertu du droit commun s'il y a lieu ${ }^{81}$. En outre, aux termes de l'article 36 bis de ce même texte, c'est toujours le juge de droit commun qui est compétent en cas d'infractions routières et d'homicide et lésions corporelles involontaires ${ }^{82}$.

Bien plus, depuis la réforme de 2006, le système belge, que d'aucuns n'hésitent plus à qualifier d'hybride ${ }^{83}$, accuse manifestement une tendance répressionnelle ${ }^{84}$ poussée, laquelle se caractérise par les procédés du procès

78 T. Moreau, “La responsabilité pénale du mineur en droit belge”, RIDP, 1 (75), (2004), pp. 151-200.

79 J. Idzuimbuir Assop, La justice pour mineurs au Zaïre: Réalités et perspectives, EUA, Kinshasa, (1990), p.19.

${ }^{80}$ F. Tulkens \& T. Moreau, F. Tulkens \& T. Moreau, Droit de la jeunesse, Larcier, Bruxelles, (2000), p.125, 162.

${ }^{81}$ C. Hennau et J. Verhaegen, Droit pénal général, Bruxelles, 1991, cité par F. TULKENS et M. Van De Kerchove, Introduction au droit pénal. Story-Scientia, Bruxelles, (1991), p. 238 et s. cités par J. Pradel, Droit pénal comparé, Dalloz, $2^{\mathrm{e}}$ édition, Paris, (2002), pp. 334-335.

${ }^{82}$ Ibid.

${ }^{83}$ Europe Ecologie Les Vert, Commission Justice, “Etat des lieux sur la justice des mineurs en Europe, Journées d'été, 08/2011, URL: http://justice.eelv.fr/etat-des-lieux-sur-la-justice-desmineurs-en-europe/, du 13/12/2014.

84 A. De Terwange, "La réforme de la loi du 08/04/1965 : à la prise en charge des mineurs ayant commis un fait qualifié d'infraction et à la réparation du dommage causé par ce fait", JDJ, $\quad \mathrm{N}^{\circ} 240, \quad$ Déc, $2004, \quad$ [en ligne] www.jdj.be/jdj/documents/docs/Reforme_loi_65_de_Terwangne_JDJD240.pdf, consulté le $24 / 05 / 15$. 
équitable $^{85}$, de dessaisissement ${ }^{86}$, de responsabilisation des mineurs par la réparation du dommage causé.

Il importe de faire état de l'avis de la section de législation du Conseil d'Etat belge sur les mesures applicables aux mineurs. En effet, dans son avis du 20 juin 1984, cette section avait affirmé que "les mesures individuelles que le tribunal de la jeunesse peut imposer au mineur... relèvent, du moins en partie, du souci de préserver la sécurité publique, au besoin par des moyens de coercition qui, en cela, s'apparentent à ceux du droit pénal ${ }^{87}$.

En réalité, Protéger-Réparer-Sanctionner sont les trois mots qui résument assez parfaitement ce système aujourd'hui. C'est dans le cadre d'une telle mutation du régime belge qu'il convient de s'interroger sur le droit congolais de l'enfant, passant du décret du 06 décembre 1950 à la loi du 09 janvier 2009.

\section{La responsabilité pénale des mineurs sous la loi du 10 janvier 2009 : Texte consacrant l'irresponsabilité pénale d'une partie d'enfants}

Ce texte avait pour principales ambitions de combler les lacunes du décret du 06 décembre 1950, de conformer le droit congolais aux standards du droit international, de réduire le fossé entre le droit et la pratique, surtout, de rapprocher les règles juridiques en cette matière aux réalités sociologiques ${ }^{88}$ congolaises. Il se présente comme un mini-code de l'enfance à l'instar de ses homologues français et belge, dont il s'inspire. Il régule la quasi-totalité des aspects juridiques de la vie du mineur en apportant des innovations sous quatre axes majeurs : le droit privé de l'enfant (droit de la personne et de la famille),

${ }^{85} \mathrm{CEDH}, \mathrm{n}^{\circ}$ 9106/80, 29/02/1988, BOUAMAR C/ Belgique.

${ }^{86}$ La gravité de l'infraction, la personnalité du mineur ou l'inefficacité avérée de mesures éducatives antérieures peuvent conduire le juge à le renvoyer devant le tribunal correctionnel ou, depuis les lois des 15/05 et 16/06/2006, devant une chambre spéciale du tribunal de la jeunesse où il sera jugé selon les règles de droit commun.

${ }^{87}$ Evoquant la question, Jean Pradel estime que cette observation s'explique par une raison constitutionnelle. En Belgique, le droit des mineurs fait partie des matières " personnalisées », soit de la compétence des communautés flamande et française, mais à l'exception des questions relevant du droit pénal (articles 459 bis et 59 ter de la Constitution), v. M. Van De Kerchove, Le droit sans peines. Aspects de la dépénalisation en Belgique et aux Etats-Unis, Bruxelles, 1987, p. 125 ; M. Van De Kerchove et F. Tulkens, Introduction au droit pénal. Aspects juridiques et criminologiques, Bruxelles, 1991, p. 229 ; cité par Jean Pradel dans son Droit pénal comparé, Op.Cit., p. 739.

$88 \mathrm{~J}$. Idzumbuir Assop, La loi sur la protection de l'enfant en RD Congo: Analyse critique et perspectives, CEDESURK, Kinshasa, (2013), p.21; R. Kienge Kienge Intudi, Le contrôle policier de la délinquance des jeunes à Kinshasa: Une approche ethnographique en criminologie, Academia Bruylant \& Kazi, Louvain-la-neuve \& Kinshasa, (2011), p.32 
le droit de la protection sociale de l'enfant, le droit judiciaire ${ }^{89}$ applicable à l'enfant et sans oublier le droit pénal de l'enfant. Cette dernière portion se pose en charnière de toute la législation. Elle consacre un droit particulier qui se subdivise en deux parties essentielles : un droit pénal des mineurs-délinquants qui prévoit des règles de fond et de forme relatives à la prise en charge des mineurs-délinquants, et un droit pénal des mineurs-victimes qui porte des circonstances aggravantes et des incriminations autonomes dont la spécificité tient du bas-âge ou du très bas-âge de la victime.

A dire vrai, la loi ${ }^{\circ} 09 / 001$ du 10 janvier 2009 portant protection de l'enfant n'irresponsabilise pas tout enfant en matière pénale. Elle n'irresponsabilise en réalité que l'une seule des deux catégories d'enfants (1). Il y a lieu de remarquer également que ce texte procède en effet plutôt par mimétisme (2), lorsqu'il organise cette responsabilité pénale, en formulant des règles purement spéciales (3), adaptées aux personnes âgées de moins de dix-huit ans.

\section{a. Une (ir)responsabilité pénale d'une partie d'enfants}

Il importe de souligner le fait que la loi de 2009 pèche, soit par excès de zèle, soit par ignorance, soit par mimétisme. Elle ne définit pas clairement ses lignes directrices. Tant et si bien qu'il semble extrêmement difficile de classer le droit congolais de l'enfant dans l'un des modèles existants. Prenant quelque chose dans presque tout, il paraît simplement inclassable et atypique.

Bien que considérée par la doctrine dominante en République démocratique du Congo comme l'aboutissement du modèle protectionniste, dont les bases auraient été posées en 1950, cette loi contient paradoxalement des notions antipodiques à ce modèle, à savoir notamment : la prohibition de prononcer seulement certaines peines choisies, l'attribution de compétence pénale au tribunal pour enfants, la subdivision de la minorité sur base du critère de discernement et la fixation d'un seuil d'irresponsabilité pénale sur cette même base (de discernement)...etc.

Absent de toutes les législations congolaises précédentes en la matière, le discernement constitue un véritable objet non identifié. Le législateur congolais l'évoque sous quatre dispositions de la loi de 2009.

- Dans les trois premières ${ }^{90}$, il reconnaît à tout enfant capable de discernement différents droits, ci-après : celui d'exprimer son opinion sur toute question l'intéressant, ses opinions étant dûment prises en

\footnotetext{
${ }^{89}$ Elle institue une juridiction spécialisée des mineurs, distincte des juridictions de droit commun, compétente pour connaître, même en appel, de toute affaire qui concerne un mineur. ${ }^{90}$ Articles 7, 32 et 33 de la loi $n^{\circ} 09 / 001$ du 10 janvier 2009 portant protection de l'enfant.
} 
considération, eu égard à son âge et à son degré de maturité ; celui d'être entendu en présence de son conseil dans toute procédure judiciaire ou administrative l'intéressant soit directement soit par l'intermédiaire d'un représentant ou d'une organisation appropriée; ainsi qu'enfin, celui d'être entendu à huis clos, en présence de son conseil, lorsqu'il est appelé à fournir des renseignements dans une procédure judiciaire. A cet effet, la capacité de discernement se trouve ici présentée comme un droit d'expression ou plutôt un droit à être consulté. Le point de vue de l'enfant ne devrait donc pas être rejeté à cause simplement de son basâge voire de son très bas-âge. Pour se prononcer sur la recevabilité ou non du point de vue de l'enfant, l'organe devant lequel il vient s'exprimer devra, au-delà de différents autres motifs qui peuvent exister selon les cas, s'assurer de la capacité ou non dudit enfant à discerner c'est-à-dire à distinguer le bien du mal. Tel que présenté dans les trois dispositions sus-évoquées, le discernement ne concerne pas particulièrement la matière pénale. Le législateur l'évoque à trois moments différents, comme suit: d'abord, il vise toute question intéressant l'enfant; ensuite, il s'intéresse précisément à la procédure judiciaire et à celle administrative ; enfin, il l'aborde en matière de procédure judiciaire simplement.

- Dans la dernière ${ }^{91}$, le discernement constitue un motif d'excuse de peine dont bénéficient les mineurs âgés de moins de 14 ans, irresponsable pénalement. C'est uniquement à l'égard de ces derniers que vaut la présomption irréfragable d'irresponsabilité, qu'organise l'article 95 de la loi de protection de l'enfant : ce qui correspond donc au seuil de l'irresponsabilité pénale avouée en faveur uniquement de cette catégorie d'enfants, en droit congolais.

Cette disposition bat complètement en brèche toute la théorie construite autour de l'irresponsabilité pénale de tous les mineurs ${ }^{92}$ en droit congolais. Puisqu'elle n'irresponsabilise nullement les mineurs âgés d'au moins 14 ans. L'interprétation, a contrario, des trois premières dispositions, démontre que la loi de protection de l'enfant reconnaît explicitement l'existence de mineurs dotés de discernement dont le régime juridique (pénal et civil) diffère, dans certains aspects, de celui de mineurs qui en sont dépourvus.

\footnotetext{
${ }^{91}$ Article 96 de la loi n ${ }^{\circ} 09 / 001$ du 10 janvier 2009 portant protection de l'enfant.

${ }^{92}$ S. Nkashama, “La loi portant protection de l'enfant : son contenu et les défis pour sa mise en œuvre", Rapport final du Séminaire international sur les droits de l'enfant en RDC, Kinshasa, 02-06/03/2009, pp. 8-9
} 
Dès lors, l'insinuation de l'existence de quelques mineurs capables de discernement, rapproche le régime congolais de 2009 à celui de ses homologues français et malien, lesquels, comparativement à lui, consacrent de manière claire, détaillée et bien réfléchie, un régime mixte, basé sur la recherche du discernement pour établir la responsabilité de type subjectif. En effet, la mixité des régimes français, et malien repose sur le mélange savamment orchestré des règles d'irresponsabilité pénale d'avec celles de responsabilité pénale des mineurs, selon les rangs et les circonstances. C'est qu'en droits français et malien, tout mineur n'est pas automatiquement absolument pénalement irresponsable. On y trouve donc des mineurs, responsables pénalement, puisque capables de discernement ; mais, (on y trouve) aussi des mineurs irresponsables pénalement, à cause de leur incapacité à discerner pour enfreindre la loi pénale.

Avec attention particulière, on se rend compte, et c'est notre humble point de vue, que c'est ce régime mixte qui caractérise aujourd'hui le droit congolais de l'enfant que porte principalement la loi $\mathrm{n}^{\circ} 09 / 001 \mathrm{du}$ 10 janvier 2009.

En conséquence de ce qui précède, un tel régime éloigne le système congolais du modèle d'irresponsabilité pénale totale de l'enfant, tant vantée, en s'appuyant sur le modèle belge ${ }^{93}$, lequel a toujours utilisé une tournure juridique consistant à coïncider le seuil de discernement à celui de la majorité pénale, de sorte à ce que la présomption d'irresponsabilité, avec ses forces et ses faiblesses, s'applique automatiquement à tous les mineurs, considérés tous non-discernants. Il s'agit là en effet plutôt d'une irresponsabilité de type objectif. En claire, ce modèle belge sous-entend la capacité de discernement à l'âge de la majorité pénale. On y est donc capable de discernement, à la condition d'être majeur d'âge pénalement. De la sorte, tout celui qui est mineur d'âge, serait incapable de discernement et automatiquement pénalement irresponsable, sur cette base essentiellement objective. Heureusement que comme explicité précédemment, les réformes intervenues tendent à fléchir cette tendance en vue de l'instauration d'une mixité.

${ }^{93}$ Lequel, avec la loi belge du 15 mai 2006, organise notamment le dessaisissement en envisageant la responsabilité pénale pour les mineurs de plus de 16 ans sous certaines conditions. Voir supra, note 28 
De ce qui précède, il convient de reconnaître que la législation congolaise d'aujourd'hui en matière d'enfants ne mérite plus d'être rangée du côté du modèle protectionniste. A cet effet, il s'impose désormais d'adopter un raisonnement adapté au contenu réel du texte actuellement en vigueur en droit congolais de l'enfant, et d'éviter d'interpréter ce texte du 10 janvier 2009 en ayant une conception ancienne, ou plutôt en recourant aux matériaux utilisés pour interpréter le décret du 06 décembre 1950 relatif à l'enfance délinquante ; encore que ce dernier n'était pas, à notre humble avis, qu'un texte d'irresponsabilisation absolue ${ }^{94}$ en matière pénale, comme pourtant dogmatiquement affirmé.

\section{b. Une responsabilité pénale optée par mimétisme}

La loi de 2009 a repris, mais certainement à sa manière, le contenu de la loi qui était censée lui servir d'inspiration. On peut se demander s'il s'agit d'un oubli, d'un silence coupable ${ }^{95}$, ou plutôt d'une option levée par le législateur congolais.

A notre humble avis, ni l'oubli, ni le silence ne trouvent bonne justification à pareille attitude. Le législateur semble avoir désormais fait son choix. En effet, notre loi de 2009 s'inspire du droit malien ${ }^{96}$ qui, à son tour connaît une forte influence du droit colonial français (art. 122-8 CP), manifestement d'obédience sanctionnelle et instituant une responsabilité subjective et graduelle dès l'âge de 13 ans. D'ailleurs, ce seuil répond aussi à la recommandation du Bureau international catholique de l'enfance ${ }^{97}$.

Mais, lorsqu'on compare notamment les articles 95 et 96 de loi congolaise de 2009 portant protection de l'enfant, d'avec l'article 98 du Code malien ${ }^{98}$ applicable à l'enfant, on est vite frappé par les "réadaptations" faites par le

\footnotetext{
${ }^{94}$ Voir supra, note 55

95 J. M. Kumbu Ki Ngimbi, “Le silence coupable”, in Afrique d'espérance, Kinshasa, n $1 \& 2$, oct. 2002-janv. 2003 \& février-mai 2003.

${ }^{96}$ La loi de protection de l'enfant est une copie surréaliste du code malien applicable à l'enfant. (Lire avec intérêt J. Idzumbuir Assop, La loi sur la protection de l'enfant en RD Congo, ..., Op.Cit., p. 63)

${ }^{97}$ Recueil sur la minorité : Analyse et commentaires de la législation applicable aux mineurs en RDC", Kinshasa, 2002, pp. 21-22, [en ligne] http://www.kirainternational.org/downloads/recueilminoriterdc.pdf, consulté le 26 février 2015

${ }^{98}$ L'article 98 du Code malien de l'enfant prévoit ce qui suit : " L'enfant âgé de moins de treize ans est présumé irréfragablement n'avoir pas la capacité d'enfreindre la loi pénale; cette présomption devient réfragable pour les enfants âgés de plus de treize ans et moins de dixhuit ans. Lorsque le prévenu ou l'accusé aura moins de treize ans, il sera relaxé ou acquitté comme ayant agi sans discernement. Lorsque le prévenu ou l'accusé aura plus de treize ans et moins de dix-huit ans, il sera relaxé ou acquitté s'il est décidé qu'il a agi sans discernement".
} 
législateur congolais. Il est moins difficile de constater l'inexistence de toute une partie du texte, supprimée volontairement par le législateur congolais, biaisant par ce fait la logique du raisonnement porté par ladite disposition malienne, sans la remettre en cause.

\section{c. Une responsabilité pénale fondée sur des règles spéciales}

De l'intitulé de la loi de 2009. Par ailleurs, pour asseoir l'idée d'une irresponsabilité pénale de l'enfant, on peut être tenté de se référer à l'intitulé de la loi du 10 janvier 2009 qui porte protection de l'enfant. Mais, il importe de noter que la protection de l'enfant ne devrait pas être prise comme synonyme à son irresponsabilité en matière pénale. Faut-il rappeler que l'intitulé d'une loi relative à la gestion pénale des mineurs n'est pas suffisant pour déterminer l'orientation effectivement prise par le législateur?

Quant à nous, le fait que la loi de 2009 soit baptisée : "loi de protection de l'enfant" ne signifie pas qu'elle irresponsabilise l'enfant. Le législateur a simplement voulu que l'enfant soit favorisé dans tous les secteurs de la vie. Qu'il bénéficie des traitements visant ou garantissant son intérêt supérieur, et cela, dans tous les domaines, y compris celui répressif. C'est ainsi qu'en matière pénale, le législateur organise un régime spécialement adapté à l'enfant. L'intitulé ne devrait donc pas désorienter les analyses. Pour nous, la protection n'est pas incompatible avec la répression. D'où, il n'est pas étonnant qu'une loi de protection organise un régime répressif propre, adaptée à la personne concernée par ladite protection.

Cette situation n'est d'ailleurs pas une particularité congolaise. On peut évoquer, à titre d'illustration le droit comparé, d'abord pour la France, l'ordonnance $\mathrm{n}^{\circ}$ 45-174 du 02 février 1945, qui confirme pourtant la justice tutélaire instaurée par la loi de 1912, mais qui porte bien l'intitulé d"' enfance délinquante"; et, même après adoption le 23 décembre 1958 (Ord. n58-1301) d'un texte dénommé de "protection de l'enfant", l'on continuait toujours à parler dans l'Hexagone des " mineurs délinquants ou dangereux". Ensuite, pour le Royaume de Belgique, le texte belge du 15 mai 1912, très semblable à son homologue français, mais brandissait déjà l'intitulé de "protection de l'enfant". Enfin, au Canada", la loi de 1908 qui consacrait l'irresponsabilité pénale des mineurs s'intitulait "loi sur les jeunes délinquants"; mais celles de 1985 puis de 2002 qui remettent en question ce principe ont pour intitulés

\footnotetext{
99 J. Trepanier, "Une justice pour mineurs en changement : Evolution nord -américaine de dernières années", RICPT, n 39-40, 1986, p.396; "La justice des mineurs au Canada: Remises en question à la fin d'un siècle", Criminologie, 32 (2), (1999), pp. 7-35, URI: http://id.erudit.org/iderudit/004749ar.
} 
respectivement "loi sur les jeunes contrevenants" et "loi sur le système de droit pénal pour adolescents".

En définitive, l'attention n'aurait pas dû être focalisée sur cette question ; puisqu'aujourd'hui tous les textes régulant la "délinquance juvénile", peu importe l'expression utilisée ou l'intitulé choisi, revendiquent toujours et déjà un système de protection, de préservation ou plutôt de garantie des droits des mineurs, construit autour du trio juge-psychologue-éducateur social et faisant appel dans bien des cas tantôt aux structures judiciaires spécialisées, tantôt aux structures extrajudiciaires.

Le défaut de pertinence de l'article 20 ter du Code pénal sur la loi de 2009. L'article 20 ter inséré par la loi n ${ }^{\circ} 15 / 022$ du 31 décembre 2015 dans le décret du 30 janvier 1940 portant Code pénal n'a apporté aucune innovation substantielle sur le débat autour de la responsabilité pénale de l'enfant; sinon à réaffirmer, dans ce texte général, le seuil minimal de responsabilité pénale en droit congolais, en reconnaissant en même temps l'absence de préjudice de cette disposition, à tout autre texte, et ici au texte spécial applicable aux mineurs. Ce nouvel article 20 ter vient certes donner un coup de jeunesse à notre cher Code pénal. Mais, si l'on se réalise qu'il y est inséré par le législateur congolais, à partir d'un texte de loi qui portait au départ, avant changement à l'Assemblée nationale, l'intitulé de "loi de mise en œuvre du statut de la Cour pénale internationale", on peut bien se rendre compte du fait que, malgré son objectif principal, il visait en réalité uniquement ce cadre bien précis ; avant le "réajustement" intervenu.

Relevons aussi que la loi $\mathrm{n}^{\circ} 15 / 022$ du 31 décembre 2015 modifiant et complétant le décret du 30 janvier 1940 portant Code pénal, introduit dans ce dernier non seulement les infractions telles que portées par le statut de Rome de la Cour pénale internationale ${ }^{100}$, mais aussi différentes causes d'irresponsabilité pénale, jadis non normatives en droit pénal congolais. Si le législateur tenait véritablement à l'irresponsabilité pénale absolue des mineurs, aucune entrave ne se serait dressée pour que la minorité figure ou soit reprise dans le paragraphe de la loi consacré aux causes d'exonération de la responsabilité pénale, pensons-nous. Curieusement et assez clairement, seuls la maladie, la déficience mentale, l'intoxication, la contrainte ou l'état

100 J. Nengowe Amundala, “ Guide pratique de la détention en RD Congo : Procédure près les juridictions de droit commun et la Cour pénale internationale", [en ligne] http://lipadhoj.org/wp-content/uploads/2016/10/EXTRAIT-Guide-pratique-de-lad\%C3\%A9tention-en-RD-Congo.pdf. 
de nécessité (avec, pour certains auteurs la légitime défense) sont repris à l'article 23 bis.

De la spécialité de la sanction applicable aux mineurs en matière pénale. Quant à la sanction, il faut relever qu'elle constitue le domaine primordial où le droit pénal des mineurs marque partout dans le monde, sa singularité.

En effet, les fonctions reconnues à la sanction pénale sont étroitement liées au fondement que les différentes doctrines pénales assignent au droit de punir. La doctrine classique lui a donné une finalité ${ }^{101}$ philosophique, morale et utilitaire, qui imprègne tout le droit pénal contemporain, en vertu duquel elle viserait à rétablir l'équilibre social, à punir le délinquant puis à le dissuader, ainsi que tous ceux qui seraient tentés de l'imiter ou de réitérer son comportement. La réadaptation ayant pris le pas sur l'élimination, la sanction pénale a intégré l'idée de traitement. Et c'est le droit pénal des mineurs qui s'est distingué bien longtemps, à imprégner, dès le début du XIX ${ }^{\text {ème }}$ siècle, la visée corrective à la sanction pénale aux moyens des mesures éducatives dont le perfectionnement et l'autonomie de plus en plus poussés remettent parfois en cause leur nature pénale. Mais, ces mesures aussi spécifiques soient-elles, ne tendent pas à la suppression du volet punitif du droit pénal des mineurs. Tout en étant répressives, elles ont pour but de privilégier, sinon de cumuler, le volet éducatif au volet punitif. Certains systèmes, comme celui du Royaume de Belgique, et celui congolais, pensons-nous, ont carrément préféré les déqualifier, en évitant de retenir leurs qualifications pénales.

D'autres par contre, le cas de la France et même de l'Allemagne, ont maintenu toutes les mesures, en retenant chaque fois les qualifications, même pénales, correspondantes. C'est ainsi qu'on y retrouve des mesures éducatives et sociales, cohabitant avec diverses peines pouvant être appliquées aux mineurs. Sans oublier aussi qu'aujourd'hui, en droit comparé comme en droit congolais, il n'est pas toujours aisé de dissocier les peines proprement dites des mesures de sureté ${ }^{102}$. D'où, on envisage une approche relativisée de la notion de sanction appliquée au mineur-délinquant.

\footnotetext{
${ }^{101}$ A. Pires, article précité ; M. Van De Kerchove, " Le sens de la peine dans la jurisprudence de la Cour suprême des USA”, RIEJ, n61, (2008); F. Tulkens \& M. Van De Kerchove, Introduction au droit pénal. Aspects juridiques et criminologiques, 8 ème éd., Bruxelles, Kluwer, (2007), pp. 528-543 ; "La nature et les contours de la peine. Regards croisés sur la jurisprudence interne et internationale", in Une criminologie de la tradition à l'innovation. En hommage à Georges Kellens, Bruxelles, Larcier, (2006), pp. 453-474.

${ }^{102}$ Likulia Bolongo, Droit pénal spécial zaïrois, LGDJ, $2^{\mathrm{ème}}$ éd., Paris, (1985), pp. 33-36 ; Droit et science pénitentiaire, LGDJ, Paris, (1981), pp. 42-44 ; NyabirungU M.S, Droit pénal général zaïrois, DES, $2^{\text {ème } e ́ d ., ~ K i n s h a s a, ~(1995), ~ p p . ~ 272-273 . ~}$
} 
L'on décèlera cette complexité aussi bien dans le décret du 06 décembre 1950 que dans la loi de protection de l'enfant de 2009 en ce qui concerne particulièrement les mesures définitives. Sous le décret, les mineurs encouraient spécifiquement des sanctions qualifiées de " mesures de garde, d'éducation et de préservation » (MGEP) dont l'une, la " mise à la disposition $d u$ gouvernement $\gg(\mathrm{MDG})$ avait explicitement le caractère d'une peine ${ }^{103}$ applicable aux majeurs d'âge.

Dans la loi de protection de l'enfant (LPE), actuellement en vigueur, et précisément dans le cadre de la médiation, le législateur prévoit la mesure de travail d'intérêt général ou de prestation communautaire (article 134). Mais, il y a lieu de remarquer que contrairement aux autres mesures prévues en ce qui concerne la médiation, cette mesure particulièrement ne vise pas directement la réparation du préjudice subi par la victime. Elle porte un caractère contraignant et vise, selon la volonté clairement exprimée du législateur, la satisfaction de la collectivité en général.

Par ailleurs, le législateur congolais adopte une attitude qui ne devrait laisser indifférent, en ce qui concerne la mesure applicable aux mineurs délinquants. - Premièrement, il n'exclut nullement la possibilité de prononcer des mesures privatives de liberté contre un mineur délinquant. Sauf qu'aux articles 10 à 12 de la loi de 2009, il soumet cette possibilité à certaines conditions, en vue de garantir l'intérêt supérieur de l'enfant qui les aura subies.

- Deuxièmement, on remarque assez facilement que ce texte (article 113 LPE) actuellement en vigueur énumère simplement un éventail de mesures, sur cinq points, dont le rattachement à un comportement et son taux sont laissés à la libre appréciation et donc au pouvoir discrétionnaire du tribunal pour enfants (TPE).

- Troisièmement, nonobstant le fait que la loi de 2009 ne cite aucune peine, le législateur interdit néanmoins, au deuxième alinéa de l'article 9 , le prononcé, à l'endroit de l'enfant, non pas de toutes les peines que le droit congolais organise ou admet en matière pénale, aux articles $5 \mathrm{du}$ décret du 30 janvier 1940 portant Code pénal, 26 de la loi nº24/2002 du

\footnotetext{
103 Articles 5 point $8^{\circ}, 14 \mathrm{~d}-14 \mathrm{j}$ du décret du 30 janvier 1940 portant Code pénal ; 26 de la loi $\mathrm{n}^{\circ} 024 / 2002$ du 18 novembre 2002 portant Code pénal militaire ; 6 in fine jusqu'à 11 du décret du 06 décembre 1950 sur l'enfance délinquante. Lire aussi K. Wembolua Otshudi, “ L'anachronisme du décret sur l'enfance délinquante en RDC : Réflexion sur le vagabondage et les droits de l'enfant', in Nouvelle tribune internationale des droits de l'enfant, $\mathrm{n}^{\circ} 12$, Mars 2007, pp. 22-28, spéc. p. 24 http://www.dei-belgique.be/IMG/pdf/reflexion sur le vagabondage et les droits de l'enfant.pdf
} 
18 novembre 2002 portant Code pénal militaire, et 77 du statut de Rome de la Cour Pénale Internationale, mais uniquement celui de deux peines les plus graves, à savoir : la peine de mort et la peine de servitude pénale à perpétuité (on peut retenir aussi la peine portant l'expression d'emprisonnement à perpétuité, organisé par le statut et ratifié par le Congo). Avec cette interdiction explicite, on ne peut qu'affirmer la non interdiction des prononcés de toutes les autres peines qui existent en droit pénal congolais. Sinon, quelle serait la portée d'une telle disposition dans un système juridique qui, en matière pénale, n'admettrait (selon une autre conception) l'application d'aucune peine aux mineurs délinquants ?

Par ailleurs, il est même prévu une mesure provisoire de privation de liberté de mouvements de l'enfant; mesure portée par l'article 108 de la loi de protection de l'enfant et s'apparentant à la détention préventive. Mais, il préfère la qualifier de placement préventif et l'admettre pour une durée ne dépassant pas deux mois.

De ce qui précède, il y a lieu de noter que le régime juridique ${ }^{104}$ auquel sont soumises toutes ces mesures spécifiques applicables aux mineurs, est loin d'être exclusivement de nature civile ou administrative. Il s'agit à n'en point douter, du moins pour certaines d'entre elles, d'un régime répressif, ou à tout le moins, d'un régime faisant appliquer les mesures ayant une forte connotation ou coloration pénale.

\section{Conclusion}

La présente réflexion a tenté de relancer le débat sur la problématique de la prise en charge des mineurs-délinquants en droit international et en droit congolais, en s'efforçant de sortir des sentiers battus et des carcans idéologiques, et en orientant, sous un autre regard, l'analyse basée sur le pragmatisme justifié par l'existence des textes au niveau international, et décriant un dogmatisme simplement affirmé en droit interne congolais.

Elle a mis en lumière les incohérences conceptuelles, idéologiques et pratiques du système congolais actuellement en vigueur, censé pourtant pallier aux insuffisances et importantes faiblesses de l'ancien, mais qui, curieusement et paradoxalement, continue à être interprété à la lumière du système qu'il doit avoir abrogé. Comme si le texte de 2009 était la copie certifiée conforme de celui de 1950, et que rien n'aurait changé. Et pourtant, pareille affirmation

${ }^{104}$ Légalité et régularité : les articles 5.2 du Décret du 06 décembre $1950 ; 10.2$, 12.2 , 113 122 de la loi de protection de l'enfant (LPE) ; 9 et 14 de la Résolution 45/113. 
rentre dans le dogmatisme injustifié et fortement décrié étant donné qu'il ne trouve aucun fondement textuel actuellement.

L'analyse a montré que l'ère tutélaire a sanctionné une nouvelle étape, la répression des comportements des mineurs-délinquants, soit clairement, la maturation des règles pénales relatives à leur délinquance. Mais, elle a également apporté à cette discipline qui existe déjà ailleurs, d'énormes controverses qui la caractérisent encore aujourd'hui et qui, pour être transcendées, appellent à des tournures, insinuations et mosaïques, parfois contre-nature. Ayant pour fil conducteur la consécration de la présomption d'innocence en faveur du mineur, les controverses qui caractérisent en République démocratique du Congo la loi n09/001 du 10 janvier 2009 sur la protection de l'enfant, en matière pénale, portent notamment sur la déqualification pénale, l'exclusivité de compétence du juge des mineurs, l'absence de consécration expresse de l'irresponsabilité pénale d'une catégorie d'enfants, la consécration d'une présomption irréfragable d'irresponsabilité en matière pénale en faveur de l'autre catégorie d'enfants, l'interdiction formelle de prononcer seulement deux peines graves en cas d'infraction commises par les enfants, savoir : la peine de mort et la servitude pénale à perpétuité...etc.

Mais, tous les moyens de ces controverses, masquant vainement la réalité, ne peuvent réfuter la nouvelle place qu'occupe à ce jour le mineur dans la société tant nationale qu'internationale; laquelle induit sa responsabilisation spéciale en matière pénale.

En effet, depuis la Convention internationale aux Droits de l'Enfant, CIDE en sigle, l'" enfant » est traité, bien que très différemment, mais comme tout être humain. Cette évolution amenuit considérablement le volet "protectionnel" pris sous l'angle d'irresponsabilisation en matière pénale.

Le discours sur "plus de droits" justifie pleinement celui de "plus de devoirs". C'est que, s'il doit bénéficier de plus de droits, le mineur doit en même temps être tenu de plusieurs devoirs. Aussi bien sur le plan international qu'interne congolais, il est dorénavant censé être apte à assumer "son enfance", mieux, sa personnalité juridique. Il est perçu comme " un acteur rationnel, équilibré, maître de ses choix et de sa destinée"105. D'où, à y regarder de près, le clivage "Mineur-Majeur" cesse d'être celui

105 H. Hamon, " L'adolescence et la justice des mineurs à l'épreuve du néolibéralisme", Adolescence, 4 (82), (2012), p. 815-822, [en ligne] http://www.cairn.info/revueadolescence-2012-4-page-815.htm 
d" incapacité-capacité" pour laisser place à celui de "capacité spéciale capacité générale".

Nous pensons quant à nous, que c'est ce qu'organise aujourd'hui, bien qu'à sa manière, le droit congolais de l'enfant, à travers sa loi du 10 janvier 2009, accusant des faiblesses nécessitant réajustements.

En définitive, à l'état actuel des textes applicables en la matière, la responsabilité pénale des mineurs trouve son fondement aussi bien dans les instruments juridiques internationaux sus-évoqués, qu'à travers ceux internes congolais.

Si le pragmatisme du droit international de la responsabilité pénale des mineurs est justifié, non seulement par sa consécration dans les instruments internationaux, sa reconnaissance, mais aussi son application ; en revanche, en droit congolais, l'étude a révélé que contrairement à l'exact contenu des textes applicables en la matière, la position adoptée depuis fort longtemps, est demeurée dogmatique et purement conjecturale. 


\section{Bibliographiques}

\section{A. Ouvrages}

Assop J, Idzuimbuir. (1990). La justice pour mineurs au Zä̈re: Réalités et perspectives. Kinshasa: EUA

Assop J, Idzuimbuir. (2013). La loi sur la protection de l'enfant en RD Congo: Analyse critique et perspectives. Kinshasa: CEDESURK

Blatier C. (2002). La délinquance des mineurs : l'enfant, le psychologue, le droit. Grenoble : PUG

Bolongo, Likulia. (1981). Droit et science pénitentiaires. Paris: LGDJ

Bonfils Ph. et Gouttenoire A. (2014). Droit des mineurs. Paris : Dalloz

David E., Tulkens F. Et Vandermeersch D. (2010). Code de droit international humanitaire. Bruylant

Intudi R, Kienge Kienge. (2011). Le contrôle policier de la délinquance des jeunes à Kinshasa: Une approche ethnographique en criminologie. Academia Bruylant \& Kazi, Louvain-la-neuve \& Kinshasa.

Matheson M. et Momtaz D. (Dir.) (2010). Les règles et institutions du droit international humanitaire à l'épreuve des conflits armés récents, Académie de droit international de la Haye.

Mouzayan O.-H. (2003). L'enfant soldat. Paris: Odile Jacob

Nerac-Croisier R. (Dir.). (1997). Le Mineur et le droit pénal. Paris : L'Harmattan

Pradel J. (2016). Droit pénal comparé. Paris : Dalloz

Pradel J. et Varinard A. (2015). Les grands arrêts du droit pénal général. Paris : Dalloz

Renucci J.F. et Courtin C. (2001). Le droit pénal des mineurs. Paris : Dalloz, $4^{\text {ème }}$ éd., col. Que sais-je,

Songa, Nyabirungu Mwene. (2007). Traité de droit pénal général congolais, Kinshasa : DES

Tulkens F. et Moreau T. (2000). Droit de la jeunesse. Bruxelles : Larcier

\section{B. Articles}

Cartuyvels Y., "Les grandes étapes de la justice des mineurs en Belgique, continuité, circularité ou ruptures?", Journal du droit des jeunes, (207), (2001).

De La Cuesta J.L., “Le nouveau statut pénal du mineur en Espagne”, RIDC, (2004), https://doi.org/10.3406/ridc.2004.19258

Dunkel Frieder, "Le droit pénal des mineurs en Allemagne: entre un système de protection et de justice", Déviance et Société, 3 (26), (2002), https://doi.org/10.3917/ds.263.0297

Melnic V., "La responsabilité pénale des mineurs dans le droit européen", Révista, 3(4), (2010) 
Moreau T., " La responsabilité pénale du mineur en droit belge", RIDP, 75 (1), (2004), https://doi.org/10.3917/ridp.751.0151.

Nkashama S., " La loi portant protection de l'enfant : son contenu et les défis pour sa mise en œuvre", Rapport final du Séminaire international sur les droits de l'enfant en RDC, Kinshasa, 02-06/03/2009

Torelli M., " Introduction à la protection internationale des droits de l'enfant", CERDIRI, (2005) 